\title{
Synthesis of Xylyl-Linked Bis-Benzimidazolium Salts and Their Application in the Palladium-Catalyzed Suzuki-Miyaura Cross-Coupling Reaction of Aryl Chlorides
}

\author{
Tsui Wang, Ting-Rong Wei, Shu-Jyun Huang, Yu-Ting Lai, Dong-Sheng Lee * ${ }^{*}$ and Ta-Jung Lu \\ Department of Chemistry, National Chung Hsing University, Taichung 40227, Taiwan; \\ s103051053@mail.nchu.edu.tw (T.W.); g107051123@mail.nchu.edu.tw (T.-R.W.); \\ g106051094@mail.nchu.edu.tw (S.-J.H.); g108051103@mail.nchu.edu.tw (Y.-T.L.); tjlu@dragon.nchu.edu.tw (T.-J.L.) \\ * Correspondence: dslee@mail.nchu.edu.tw; Tel.: +886-4-22862547
}

check for

updates

Citation: Wang, T.; Wei, T.-R.;

Huang, S.-J.; Lai, Y.-T.; Lee, D.-S.; Lu,

T.-J. Synthesis of Xylyl-Linked

Bis-Benzimidazolium Salts and Their

Application in the

Palladium-Catalyzed

Suzuki-Miyaura Cross-Coupling

Reaction of Aryl Chlorides. Catalysts

2021, 11, 817. https://doi.org/

$10.3390 /$ catal11070817

Academic Editor: Atsushi Ohtaka

Received: 18 June 2021

Accepted: 3 July 2021

Published: 5 July 2021

Publisher's Note: MDPI stays neutral with regard to jurisdictional claims in published maps and institutional affiliations.

Copyright: (c) 2021 by the authors. Licensee MDPI, Basel, Switzerland. This article is an open access article distributed under the terms and conditions of the Creative Commons Attribution (CC BY) license (https:/ / creativecommons.org/licenses/by/ $4.0 /)$.

\begin{abstract}
A new series of xylyl-linked bis-benzimidazolium salts were efficiently prepared using a simple preparation method from bis-benzimidazolium precursors featuring highly tunable linkers and wingtips. A highly efficient Suzuki-Miyaura cross-coupling reaction of aryl chlorides within the range of $0.5-2.0 \mathrm{~mol} \% \mathrm{Pd}$-catalyst loading was observed. Also, di-ortho-substituted biaryl synthesis was achieved.
\end{abstract}

Keywords: bis-benzimidazolium salt; coupling reaction; aryl chloride; di-ortho-substituted biaryl; $N$-heterocyclic carbene

\section{Introduction}

The palladium-catalyzed Suzuki-Miyaura cross-coupling reaction (SMC), which involves the combination of an aryl halide with an arylboronic acid in the presence of a Pd catalyst, is one of the most practical protocols by which to achieve $C-C$ bond formation [1-5]. Aryl chlorides, which are less expensive and more diverse than aryl bromides and iodides, are challenging compounds to use in SMC reactions due to their low $\mathrm{C}-\mathrm{Cl}$ bond reactivity [6]. Numerous successful examples using a combination of phosphine ligands with $\mathrm{Pd}$ have been reported, however, the disadvantages of using such ligands are their difficult preparation, air-sensitivity, expense, and toxicity [7-9]. The development of Pd catalysts with phosphine-free ligands has thus received a great amount of attention.

Over the past three decades, the replacement of phosphine ligands with $N$-heterocyclic carbenes (NHCs) has been shown to be a good choice. Compared with bulky tertiary phosphines, these new NHC ligands exhibit strong $\sigma$-donor but poor $\pi$-acceptor properties [10]. Metal-NHC complexes exhibit extraordinary heat, air, and moisture stability due to the high dissociation energies of their metal-carbon bonds [11]. Bis-NHC binds to metals to form more stable complexes compared to monodentate NHC complexes, which has led to the development of such ligands attracting great attention in the literature. In addition, the diversity of bis-NHC compounds is also an advantage, as they have tunable linkers and wingtips. In 1998, a chelating bis-NHC ligand was synthesized by Herrmann and co-workers [12] and applied in the Pd-catalyzed SMC reaction of 4-chloroacetophenone and phenylboronic acid at $120^{\circ} \mathrm{C}$ for $48 \mathrm{~h}$, resulting in the desired product being formed with a yield of $60 \%$. Numerous successful examples of SMC reactions between aryl chlorides and arylboronic acids employing Pd-bis-NHC complexes or in-situ-formed $\mathrm{Pd}(\mathrm{OAc})_{2} /$ bisNHC catalyst systems have been previously reported [13-29]. However, high catalyst loading and the variety of the substrates still present challenge. 
The catalytic reaction rate is affected by the nature of the NHC ligand. To obtain efficient bis-NHC ligands, a series of new xylyl-linked bis-benzimidazolium salts was prepared by modifying the wingtips and linkers of a bis-NHC precursor. These salts have the following advantages: (1) our linker contains aryl group which can provide electronic effect, (2) our linker has an additional methylene group compared to currently available NHCs in which the aryl group is directly connected to the bis-benzimidazole moiety that offers more conformational flexibility, and (3) the wingtip delivers electronic and steric effects. Those advantages facilitate the oxidative addition and reductive elimination steps of the SMC reaction. We report herein their application in an in-situ-generated $\mathrm{Pd}(\mathrm{OAc})_{2} /$ bis-NHC catalyst system for the SMC reaction of aryl chlorides with arylboronic acids (Figure 1).
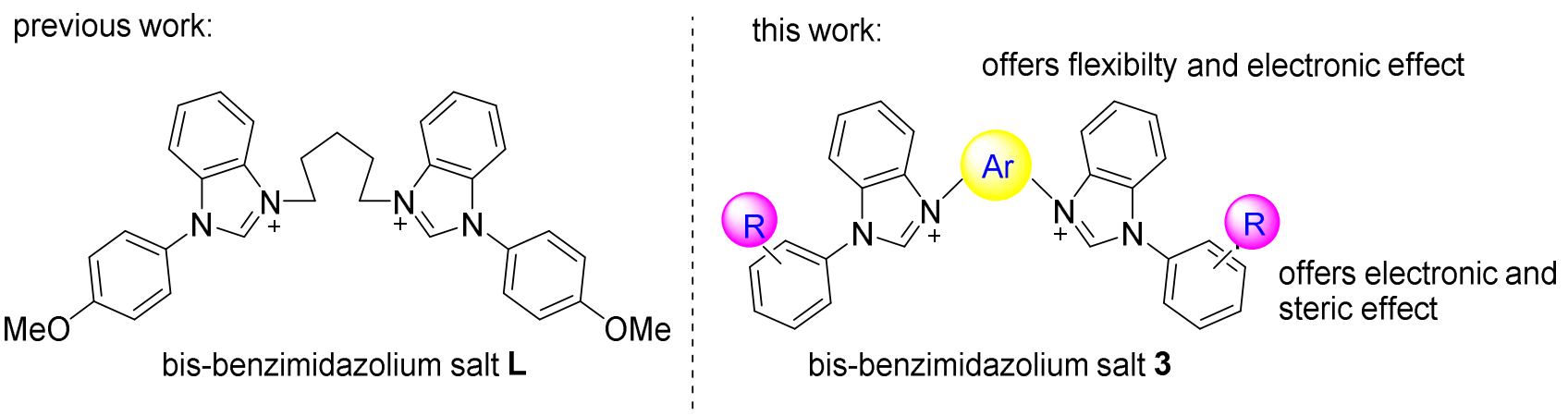

Figure 1. The structures of the new xylyl-linked bis-benzimidazolium salts 3 .

\section{Results}

\subsection{Synthesis and Characterization of the Bis-Benzimidazolium Salts 3}

According to Scheme 1, the bis-benzimidazolium salts 3 were prepared by the combination of two equivalents of the corresponding $N$-arylbenzimidazole 2 and one equivalent of the desired dibromides 1 in acetonitrile under reflux to produce the respective products with yields in the range of 50 to $89 \%$. The newly prepared salts were found to be air- and moisture-stable both in the solid state and in solution, and were characterized by ${ }^{1} \mathrm{H}$ and ${ }^{13} \mathrm{C}$ nuclear magnetic resonance (NMR) spectroscopy.

In the ${ }^{1} \mathrm{H}$ NMR spectra of $\mathbf{3 a}-\mathbf{3 d}$, the methylene protons could be observed as a singlet, while the ${ }^{1} \mathrm{H}$ NMR spectra of $3 \mathbf{e}$ and $3 \mathbf{f}$ showed an $\mathrm{AB}$ spin system for the methylene protons. The benzimidazolium proton signals in the spectra of $3 \mathbf{a}-3 \mathbf{f}$ could be observed in a wide range of between $\delta 10.15$ and $11.57 \mathrm{ppm}$. The ${ }^{13} \mathrm{C}$ NMR spectra of $\mathbf{3 a}-\mathbf{3} \mathbf{f}$ showed the NCHN resonances between $\delta 142.4$ and $161.0 \mathrm{ppm}$. 
<smiles>[X]c1cccc(CBr)c1</smiles>

$1 \mathrm{a}=\mathrm{X}=\mathrm{CH}$ $1 b=X=N$<smiles>[Ga]n1cnc2ccccc21</smiles>

$2 \mathrm{a}=\mathrm{Ar}=4-\mathrm{MeO}-\mathrm{C}_{6} \mathrm{H}_{4}$ $\mathbf{2 b}=\mathrm{Ar}=2,4,6-\mathrm{Me}_{3}-\mathrm{C}_{6} \mathrm{H}_{2}$<smiles></smiles>

$2 \overline{B r}$

$3 \mathbf{a}=\mathrm{Ar}=4-\mathrm{MeO}-\mathrm{C}_{6} \mathrm{H}_{4}, \mathrm{X}=\mathrm{CH} ; 88 \%$

$3 \mathrm{~b}=\mathrm{Ar}=$ Mesyl, $\mathrm{X}=\mathrm{CH} ; 89 \%$

$3 \mathrm{c}=\mathrm{Ar}=4-\mathrm{MeO}-\mathrm{C}_{6} \mathrm{H}_{4}, \mathrm{X}=\mathrm{N} ; 82 \%$

$3 d=A r=$ Mesityl, $X=N ; 50 \%$

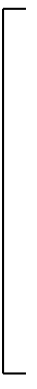<smiles></smiles>

$3 \mathrm{e}=\mathrm{Ar}=4-\mathrm{MeO}-\mathrm{C}_{6} \mathrm{H}_{4} ; 78 \%$

$3 \mathrm{f}=\mathrm{Ar}=$ Mesityl; $68 \%$

Scheme 1. Overview of the synthesis of bis-benzimidazolium salts 3 .

\subsection{The Suzuki-Miyaura Cross-Coupling Reaction}

Continuing our previous studies on the application of in-situ-formed catalyst systems for the SMC reaction [28,29], 4-chloroacetophenone $\mathbf{4 a}$ and phenylboronic acid 5a were used as model starting materials (Table 1$)$. Under classical conditions $\left(\mathrm{K}_{3} \mathrm{PO}_{4} \cdot \mathrm{H}_{2} \mathrm{O}, \mathrm{Pd}(\mathrm{OAc})_{2}\right.$, $t$-BuOH, $\left.30{ }^{\circ} \mathrm{C}, 24 \mathrm{~h}\right)$, the activity of the $\mathrm{L} / \mathrm{Pd}(\mathrm{OAc})_{2}$ catalyst system was first compared with that of $3 \mathbf{b} / \mathrm{Pd}(\mathrm{OAc})_{2}$ (Table 1 , entries 1 and 2$)$. It was found that the xylyl-bridged bis-benzimidazolium precursor $\mathbf{3 b}$ showed better activity in comparison to alkyl-linked bis-benzimidazolium salt $\mathbf{L}$ (Table 1 , entries 1 and 2). Next, the activity of the $3 / \mathrm{Pd}(\mathrm{OAc})_{2}$ catalyst systems was compared. Upon increasing the reaction temperature to $80{ }^{\circ} \mathrm{C}$, the gas chromatography (GC) yield of 6aa increased from $21 \%$ to $88 \%$ (Table 1, entries 2 and 3 ). The focus was shifted to investigate the influence that the xylyl-linked spacers and wingtips have on the benzimidazole ring. With the same wingtip on the benzimidazole ring (3b, 3d, and 3f), the bis-benzimidazolium precursor with a benzene ring as a xylyllinked spacer showed better activity in comparison to when pyridine or biphenyl were incorporated in the xylyl-linked bridge bis-benzimidazolium salts (Table 1, entries 3, 6, and 8). The same results were also observed using bis-benzimidazolium salts $\mathbf{3 a}, \mathbf{3} \mathbf{c}$, and $\mathbf{3 e}$ (Table 1 , entries 4,5 , and 7 ). The activity of the bis-benzimidazolium salts with a mesityl group on the benzimidazole ring as the wingtip (3d and $\mathbf{3 f}$ ) was better than that when a methoxyphenyl group was incorporated as a wingtip (3c and 3e), regardless of whether pyridine or biphenyl was present as the xylyl-linked bridge in the bis-benzimidazolium salts (Table 1, entries 5-8). However, both $\mathbf{3 a}$ and $\mathbf{3 b}$ exhibited similar catalytic activity (Table 1, entries 3 and 4), an observation that shows that having a mesityl group on the benzimidazole ring leads to a sterically hindered effect, which is beneficial in the reductive elimination step of the catalytic cycle. 
Table 1. Screening the activity of the in-situ-formed bis-NHC/Pd catalyst ${ }^{1}$.

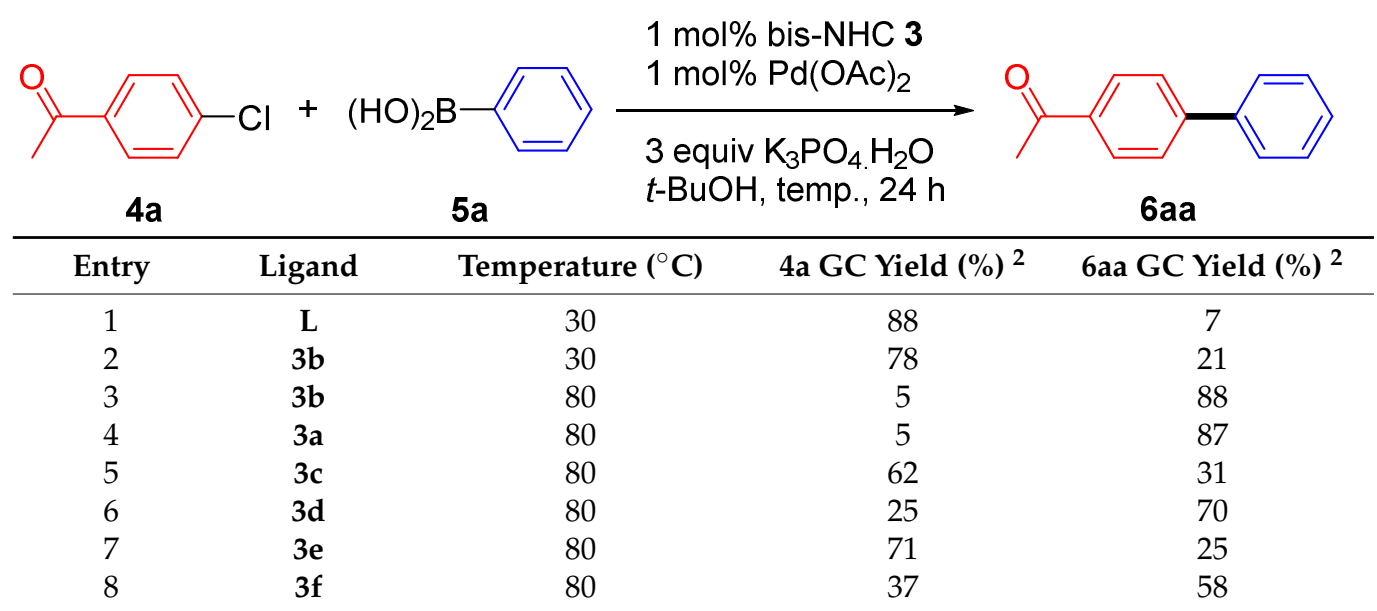

${ }^{1}$ Reaction conditions: 4a $(1.0 \mathrm{mmol}), \mathbf{5 a}(1.5 \mathrm{mmol})$, bis-NHC $\left.3(1 \mathrm{~mol} \%), \mathrm{Pd}(\mathrm{OAc})\right)_{2}(1 \mathrm{~mol} \%), \mathrm{K}_{3} \mathrm{PO}_{4} \cdot \mathrm{H}_{2} \mathrm{O}$ $(3.0 \mathrm{mmol})$ in $t-\mathrm{BuOH}(3.0 \mathrm{~mL})$ for $24 \mathrm{~h}$ under a $\mathrm{N}_{2}$ atmosphere. ${ }^{2}$ Determined by gas-chromatography-flame ionization detection (GC-FID) using undecane as an internal standard.

The effects of different solvents, bases, and Pd loading amounts on the catalytic activity of this reaction are summarized in Table 2. Most of the solvents tested gave the products in good to excellent yields (87-94\%, Table 2, entries 1-4) in this work, except for a mixed solvent of $t$-butanol $(\mathrm{BuOH}) / \mathrm{H}_{2} \mathrm{O}$, the use of which led to a poor product yield (55\%, Table 2, entry 5). It was observed that 1,4-dioxane was the best choice of solvent for producing products with higher conversion rates (Table 2, entry 2). The replacement of $\mathrm{K}_{3} \mathrm{PO}_{4} \cdot \mathrm{H}_{2} \mathrm{O}$ with $\mathrm{KO}^{t} \mathrm{Bu}$ or $\mathrm{KOAc}$ led to lower $\mathrm{GC}$ yields of the respective products (Table 2, entries 6 and 9) and when $\mathrm{K}_{2} \mathrm{CO}_{3}$ or $\mathrm{Cs}_{2} \mathrm{CO}_{3}$ was used instead, excellent yields were observed (Table 2, entries 7 and 8). After screening Pd loading amount, the coupling reaction could be carried out in the presence of $0.5 \mathrm{~mol} \% \mathrm{Pd}(\mathrm{OAc})_{2} / 3 \mathrm{~b}$ catalyst for $6 \mathrm{~h}$ in $98 \%$ yield (Table 2 , entry 13 ).

Table 2. Optimization of reaction conditions ${ }^{1}$.

\begin{tabular}{|c|c|c|c|c|c|c|}
\hline Entry & $3 \mathrm{~b} / \mathrm{Pd}(\mathrm{OAc})_{2}(\mathrm{~mol} \%)$ & Solvent & Base & Time (h) & 4a GC Yield (\%) ${ }^{2}$ & 6aa GC Yield (\%) ${ }^{2}$ \\
\hline 1 & 1.0 & Toluene & $\mathrm{K}_{3} \mathrm{PO}_{4} \cdot \mathrm{H}_{2} \mathrm{O}$ & 24 & 0 & $91(91)^{3}$ \\
\hline 2 & 1.0 & 1,4-Dioxane & $\mathrm{K}_{3} \mathrm{PO}_{4} \cdot \mathrm{H}_{2} \mathrm{O}$ & 24 & 0 & $94(94)^{3}$ \\
\hline 3 & $1.0^{4}$ & 1,4-Dioxane & $\mathrm{K}_{3} \mathrm{PO}_{4} \cdot \mathrm{H}_{2} \mathrm{O}$ & 24 & - & $(20)^{3}$ \\
\hline 4 & 1.0 & $\mathrm{CH}_{3} \mathrm{CN}$ & $\mathrm{K}_{3} \mathrm{PO}_{4} \cdot \mathrm{H}_{2} \mathrm{O}$ & 24 & 7 & 87 \\
\hline 5 & 1.0 & $t-\mathrm{BuOH}$ & $\mathrm{K}_{3} \mathrm{PO}_{4} \cdot \mathrm{H}_{2} \mathrm{O}$ & 24 & 5 & 88 \\
\hline 6 & 1.0 & $t-\mathrm{BuOH} / \mathrm{H}_{2} \mathrm{O}^{5}$ & $\mathrm{~K}_{3} \mathrm{PO}_{4} \cdot \mathrm{H}_{2} \mathrm{O}$ & 24 & 31 & 55 \\
\hline 7 & 1.0 & 1,4-Dioxane & $\mathrm{KO}^{t} \mathrm{Bu}$ & 24 & 1 & 4 \\
\hline 8 & 1.0 & 1,4-Dioxane & $\mathrm{K}_{2} \mathrm{CO}_{3}$ & 24 & 13 & 82 \\
\hline 9 & 1.0 & 1,4-Dioxane & $\mathrm{Cs}_{2} \mathrm{CO}_{3}$ & 24 & - & $(93)^{3}$ \\
\hline 10 & 1.0 & 1,4-Dioxane & KOAc & 24 & 86 & 8 \\
\hline 11 & 1.0 & 1,4-Dioxane & $\mathrm{K}_{3} \mathrm{PO}_{4} \cdot \mathrm{H}_{2} \mathrm{O}$ & 12 & 0 & 96 \\
\hline 12 & 1.0 & 1,4-Dioxane & $\mathrm{K}_{3} \mathrm{PO}_{4} \cdot \mathrm{H}_{2} \mathrm{O}$ & 6 & 0 & $99(98)^{3}$ \\
\hline 13 & 1.0 & 1,4-Dioxane & $\mathrm{K}_{3} \mathrm{PO}_{4} \cdot \mathrm{H}_{2} \mathrm{O}$ & 3 & 60 & 35 \\
\hline 14 & 0.5 & 1,4-Dioxane & $\mathrm{K}_{3} \mathrm{PO}_{4} \cdot \mathrm{H}_{2} \mathrm{O}$ & 6 & 0 & $99(98)^{3}$ \\
\hline 15 & 0.1 & 1,4-Dioxane & $\mathrm{K}_{3} \mathrm{PO}_{4} \cdot \mathrm{H}_{2} \mathrm{O}$ & 6 & 65 & 30 \\
\hline 16 & 0.05 & 1,4-Dioxane & $\mathrm{K}_{3} \mathrm{PO}_{4} \cdot \mathrm{H}_{2} \mathrm{O}$ & 6 & 90 & 0.4 \\
\hline
\end{tabular}

${ }^{1}$ Reaction conditions: $\mathbf{4 a}(1.0 \mathrm{mmol}), \mathbf{5 a}(1.5 \mathrm{mmol}), \mathbf{3 b} / \mathrm{Pd}(\mathrm{OAc})_{2}(\mathrm{x} \mathrm{mol} \%, \mathbf{3 b}: \mathrm{Pd}=1: 1)$, base $(3.0 \mathrm{mmol})$ in solvent $(3.0 \mathrm{~mL})$ under a $\mathrm{N}_{2}$ atmosphere. ${ }^{2}$ Determined by GC-FID using undecane as an internal standard. ${ }^{3}$ Isolated yield in parentheses. ${ }^{4} \mathrm{~L} / \mathrm{Pd}(\mathrm{OAc})_{2}$ was used. The reaction was carried out at $110{ }^{\circ} \mathrm{C} .{ }^{5} t-\mathrm{BuOH} / \mathrm{H}_{2} \mathrm{O}=6 / 4(\mathrm{vol} / \mathrm{vol})$.

The scope of the SMC reaction was studied after screening the optimized reaction conditions (Table 3). The coupling reactions of aryl chlorides $\mathbf{4 a}, \mathbf{4 b}$, and $\mathbf{4 c}$ with electronwithdrawing groups proceeded in quantitative yields in line with an increase in the catalyst 
loading and temperature (6aa-6ca). Similarly, the SMC reaction was carried out at $80{ }^{\circ} \mathrm{C}$ in the presence of the catalyst with $1.0 \mathrm{~mol} \%$ loading using $m$-chloropropiophenone and $p$-chloropropiophenone as starting materials and quantitative yields of the desired products 6da and 6ea were obtained. With other electron-withdrawing groups at the para position, such as in $4 \mathbf{f}$ and $4 \mathbf{i}, 96 \%$ and $90 \%$ isolated yields were obtained, respectively (6fa and $6 \mathbf{i a})$, whereas, the coupling reactions of 1-chloro-2-nitrobenzene, $4 \mathrm{~g}$, and 1-chloro-2,4dinitrobenzene, $4 \mathbf{h}$, with phenylboronic acid showed good yields $(81-89 \%)$ at $100{ }^{\circ} \mathrm{C}$. Aryl chlorides $(4 \mathbf{a}, 4 \mathrm{~d}, 4 \mathbf{e}, \mathbf{4 f}$, and $4 \mathbf{i})$ bearing electron-withdrawing groups at their $m$ - or $p$ positions were coupled with 1-naphthylboronic acid at $100{ }^{\circ} \mathrm{C}$ in the presence of a catalyst with $1.5 \mathrm{~mol} \%$ loading to give the respective products in yield of $90-99 \%(6 \mathbf{a b}-6 \mathbf{i b})$. It was observed that the coupling reactions of benzyl chloride with phenylboronic acid or 1-naphthylboronic acid led to $95 \%$ and $99 \%$ yields of $\mathbf{6 j a}$ and $\mathbf{6 j b}$, respectively, under the same reaction conditions. The aryl chlorides $4 \mathbf{k}$ and $4 \mathbf{l}$ with electron-donating groups gave the corresponding products (6ka and 6la) in poor yields. Interestingly, $o$-chlorotoluene was coupled with phenylboronic acid in the presence of a $1 \mathrm{~mol} \% 3 \mathbf{b} / \mathrm{Pd}(\mathrm{OAc})_{2}$ catalyst at $80^{\circ} \mathrm{C}(6 \mathrm{ma})$ to give the desired product in $95 \%$ yield. Finally, the di-ortho-substituted biaryl syntheses of $o$-nitroarenes ( $4 \mathrm{n}$ and $4 \mathrm{~g}$ ) with 1-naphthylboronic acid (5b) led to excellent yields of the respective products, with a $93 \%$ yield of $6 \mathbf{h b}$ and a $92 \%$ yield of $6 \mathrm{gb}$ observed.

Table 3. In-situ-generated $3 b / \mathrm{Pd}(\mathrm{OAc})_{2}$ catalyst catalyzed Suzuki-Miyaura cross-coupling reactions ${ }^{1}$.
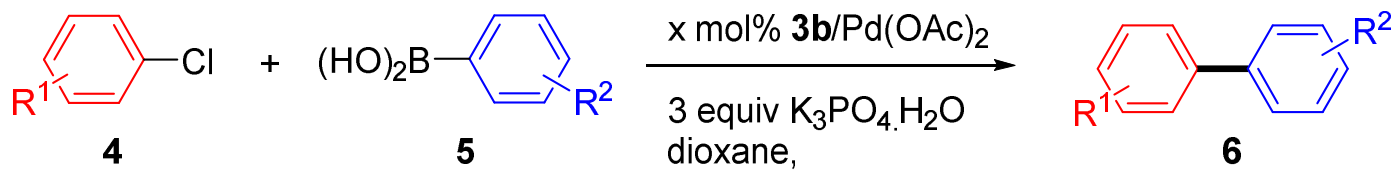

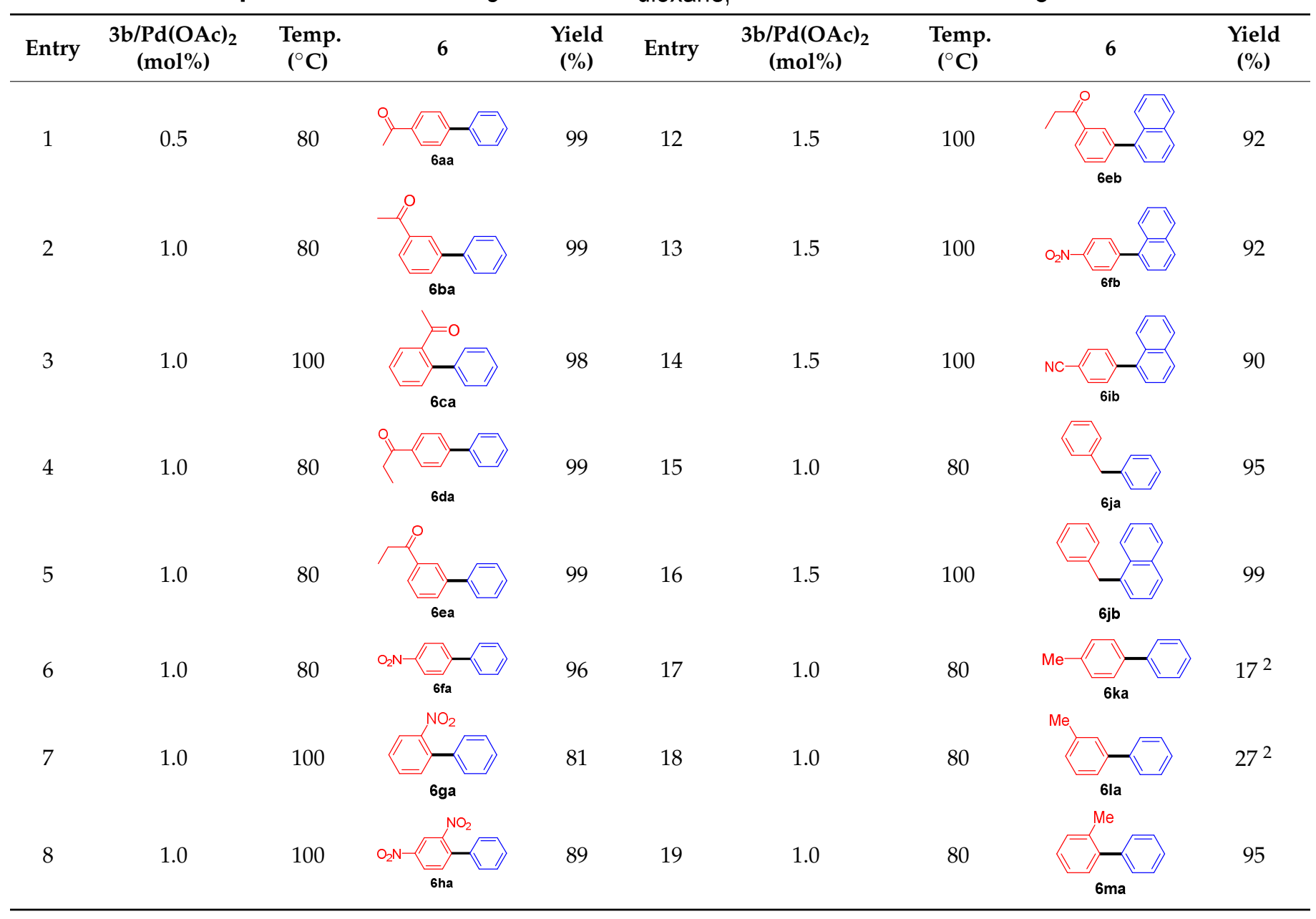


Table 3. Cont.

\begin{tabular}{|c|c|c|c|c|c|c|c|c|}
\hline Entry & $\begin{array}{c}3 \mathrm{~b} / \mathrm{Pd}(\mathrm{OAc})_{2} \\
(\mathrm{~mol} \%)\end{array}$ & $\begin{array}{l}\text { Temp. } \\
\left({ }^{\circ} \mathrm{C}\right)\end{array}$ & 6 & $\begin{array}{c}\text { Yield } \\
(\%)\end{array}$ & Entry & $\begin{array}{c}3 \mathrm{~b} / \mathrm{Pd}(\mathrm{OAc})_{2} \\
(\mathrm{~mol} \%)\end{array}$ & $\begin{array}{l}\text { Temp. } \\
\left({ }^{\circ} \mathrm{C}\right)\end{array}$ & $\begin{array}{c}\text { Yield } \\
(\%)\end{array}$ \\
\hline 9 & 1.0 & 80 & $6 i$ & 90 & 20 & 2.0 & 100 & 93 \\
\hline 10 & 1.5 & 100 & $6 a b$ & 99 & 21 & 2.0 & 100 & 92 \\
\hline 11 & 1.5 & 100 & 6 & 97 & & & & \\
\hline
\end{tabular}

${ }^{1}$ Reaction conditions: 4 (1.0 mmol), 5 (1.5 mmol), 3b/Pd(OAc) $)_{2}(x \mathrm{~mol} \%, 3 \mathbf{b}: \mathrm{Pd}=1: 1), \mathrm{K}_{3} \mathrm{PO}_{4} \cdot \mathrm{H}_{2} \mathrm{O}(3.0 \mathrm{mmol})$ in 1,4-dioxane (3.0 mL) under a $\mathrm{N}_{2}$ atmosphere. Isolated yield was reported. ${ }^{2}$ GC-FID yield was reported by using undecane as an internal standard.

\section{Materials and Methods}

\subsection{General Methods}

Unless otherwise stated, commercially available materials were received from Aldrich and Acros and used without further purification. Acetonitrile was distilled over calcium hydride prior to its use. Toluene, 1,4-dioxane, and $t$ - $\mathrm{BuOH}$ were distillated over sodium prior to its use. Reactions were monitored using pre-coated silica gel 60 (F-254) plates. The products were purified by column chromatography (silica gel, 0.040-0.063 $\mu \mathrm{m}$ ), eluting with $n$-hexane/ethyl acetate. ${ }^{1} \mathrm{H}$ and ${ }^{13} \mathrm{C}$ NMR spectra were recorded using an Agilent Mercury 400 spectrometer(Agilent Technologies Inc., Santa Clara, CA, USA), with the $J$-values given in $\mathrm{Hz}$. Chemical shifts $(\delta)$ were referenced to $\mathrm{CDCl}_{3}(\delta=7.26 \mathrm{ppm})$ in the ${ }^{1} \mathrm{H}$ NMR spectra and $\mathrm{CDCl}_{3}(\delta=77.0 \mathrm{ppm})$ in the ${ }^{13} \mathrm{C}$ NMR spectra. Copies of ${ }^{1} \mathrm{H}$ and ${ }^{13} \mathrm{C}$ NMR spectra of all compounds are provided as Supplementary Materials. Melting points were determined using Thermo 1001D digital melting point apparatus and are uncorrected. GC-FID was recorded using a Shimadzu GC-2014 spectrometer (Shimadzu Co., Kyoto, Japan) equipped with a capillary column $\left(\mathrm{SPB}^{\circledR}{ }^{\circledR}-5,60 \mathrm{~m} \times 0.25 \mathrm{~mm} \times 0.25 \mu \mathrm{m}\right)$. The conversion yields, GC yields, and ratios were determined using undecane as an internal standard. High-resolution mass spectra were recorded using a Finnigan/Thermo Quest MAT 95XL mass spectrometer (Finnigan MAT LCQ, San Jose, CA, USA) via either electron impact (EI) or electrospray ionization (ESI) methods. 1,3-Bis-(bromomethyl)benzene 1a [30], 2,6-bis-(bromomethyl)pyridine 1b [31], 2,2'-bis-(bromomethyl)benzene 1c [32], and 1-(4methoxyphenyl)-1H-benzo[d]imidazole 2a [29] were synthesized according to modified literature procedures.

\subsection{Experimental Procedures and Spectral Data}

3.2.1. Synthesis of 1-(2,4,6-Trimethylphenyl)-1H-benzimidazole $\mathbf{2 b}$

Procedure for 2-(2', $4^{\prime}, 6^{\prime}$-trimethylanilido)nitrobenzene (S1) [33]. A sealed tube was charged with 2-fluoronitrobenzene $(0.15 \mathrm{~mL}, 1.43 \mathrm{mmol})$, 2,4,6-trimethylaniline $(0.30 \mathrm{~mL}, 2.14 \mathrm{mmol})$, and anhydrous potassium fluoride $(0.08 \mathrm{~g}, 1.43 \mathrm{mmol})$. The reaction mixture was heated at $180{ }^{\circ} \mathrm{C}$ for $48 \mathrm{~h}$. After completion of the reaction (monitored by TLC), the mixture was cooled to room temperature and then quenched with water $(15 \mathrm{~mL})$. The aqueous phase was extracted with EtOAc $(30 \mathrm{~mL} \times 3)$. The combined organic layers were dried over $\mathrm{MgSO}_{4}$ and then filtered. The solvent was evaporated under reduced pressure to afford crude product which was purified by chromatography to afford S1 as an orange solid $(0.35 \mathrm{~g}, 97 \%) .{ }^{1} \mathrm{H}$ NMR $\left(\mathrm{CDCl}_{3}, 400 \mathrm{MHz}\right): \delta 9.12(\mathrm{~s}, 1 \mathrm{H}, \mathrm{NH}), 8.22(\mathrm{~d}, J=7.6 \mathrm{~Hz}, 1 \mathrm{H}), 7.27$ $(\mathrm{t}, J=7.6 \mathrm{~Hz}, 1 \mathrm{H}), 7.00(\mathrm{~s}, 2 \mathrm{H}), 6.68(\mathrm{t}, J=7.6 \mathrm{~Hz}, 1 \mathrm{H}, 6.38(\mathrm{~d}, J=8.8 \mathrm{~Hz}, 1 \mathrm{H}), 2.33(\mathrm{~s}, 3 \mathrm{H})$, 
2.15 (s, 6H); ${ }^{13} \mathrm{C} \mathrm{NMR}\left(\mathrm{CDCl}_{3}, 100 \mathrm{MHz}\right): \delta 144.7,137.3,136.3,136.1,132.6,131.9,129.4$, $126.7,116.2,115.0,21.0,18.1$.

Procedure for $\mathrm{N}-\left(2^{\prime}, 4^{\prime}, 6^{\prime}\right.$-trimethylphenyl)-1,2-phenylenediamine (S2) [33] ${ }^{5}$ A $250 \mathrm{~mL}$ round-bottom flask was charged with 2-(2', $4^{\prime}, 6^{\prime}$-trimethylanilido)nitrobenzene $\mathbf{S 1}$ ( $2.18 \mathrm{~g}$, $8.49 \mathrm{mmol})$ and ethanol $(60 \mathrm{~mL})$. The reaction mixture was heated at $60{ }^{\circ} \mathrm{C}$. After the 2-(2', $4^{\prime}, 6^{\prime}$-trimethylanilido)nitrobenzene was dissolved completely, the solution was added ammonium chloride solution $\left(0.125 \mathrm{~g} / 1 \mathrm{~mL} \mathrm{H}_{2} \mathrm{O}, 30 \mathrm{~mL}, 85.0 \mathrm{mmol}\right)$, and iron $(4.74 \mathrm{~g}$, $85.0 \mathrm{mmol}$ ). The reaction mixture was refluxed for $1.5 \mathrm{~h}$. After completion of the reaction (monitored by TLC), solvent was removed under reduced pressure, the residue was added with $\mathrm{H}_{2} \mathrm{O}(3 \mathrm{~mL})$. The aqueous layer was extracted with EtOAc $(3 \mathrm{~mL} \times 3)$. The combined organic layers were dried over $\mathrm{MgSO}_{4}$ and then filtered. The solvent was evaporated under reduced pressure to afford $\mathbf{S 2}$ as a deep purple solid $(1.59 \mathrm{~g}, 99 \%) .{ }^{1} \mathrm{H} \mathrm{NMR}\left(\mathrm{CDCl}_{3}\right.$, $400 \mathrm{MHz}): \delta 6.93(\mathrm{~s}, 2 \mathrm{H}), 6.79(\mathrm{~d}, J=7.2 \mathrm{~Hz}, 1 \mathrm{H}), 6.74(\mathrm{t}, J=7.2 \mathrm{~Hz}, 1 \mathrm{H}), 6.64(\mathrm{t}, J=7.2 \mathrm{~Hz}$, $1 \mathrm{H}), 6.23(\mathrm{~d}, J=7.2 \mathrm{~Hz}, 1 \mathrm{H}), 4.76(\mathrm{~s}, 1 \mathrm{H}, \mathrm{NH}), 3.63(\mathrm{~s}, 2 \mathrm{H}, \mathrm{NH}), 2.30(\mathrm{~s}, 3 \mathrm{H}), 2.12(\mathrm{~s}, 6 \mathrm{H}) ;{ }^{13} \mathrm{C}$ NMR (CDCl $3,100 \mathrm{MHz}): \delta 136.9,135.6,134.7,133.9,133.3,129.3,120.2,120.0,116.2,114.9$, 20.8, 18.0.

Procedure for 1-(2,4,6-trimethylphenyl)-1H-benzimidazole (2b) [34] ${ }^{6}$. To a solution of $\mathrm{N}-\left(2^{\prime}, 4^{\prime}, 6^{\prime}\right.$-trimethylphenyl)-1,2-phenylenediamine S2 $(0.40 \mathrm{~g}, 1.8 \mathrm{mmol})$ and sulfamic acid $(19.0 \mathrm{mg}, 0.20 \mathrm{mmol})$ in methanol $(10 \mathrm{~mL})$ was added triethyl orthoformate $(1.2 \mathrm{~mL}$, $6.9 \mathrm{mmol}$ ) at rt. After stirring overnight at $\mathrm{rt}$ (monitored by TLC), methanol was removed by rotary evaporator. $\mathrm{H}_{2} \mathrm{O}(3 \mathrm{~mL})$ was added to the residue. The aqueous layer was extracted with EtOAc $(3 \mathrm{~mL} \times 3)$. The combined organic layers were dried over $\mathrm{MgSO}_{4}$ and then filtered. The solvent was evaporated under reduced pressure to afford crude product which was purified by chromatography to afford $\mathbf{2 b}$ as a deep purple solid $(0.37 \mathrm{~g}$, 90\%). ${ }^{1} \mathrm{H} \mathrm{NMR}\left(\mathrm{CDCl}_{3}, 400 \mathrm{MHz}\right): 87.89(\mathrm{~d}, J=8.0 \mathrm{~Hz}, 1 \mathrm{H}), 7.87(\mathrm{~s}, 1 \mathrm{H}), 7.32(\mathrm{t}, J=7.2 \mathrm{~Hz}$, $1 \mathrm{H}), 7.26(\mathrm{t}, J=7.2 \mathrm{~Hz}, 1 \mathrm{H}), 7.05-7.02(\mathrm{~m}, 3 \mathrm{H}), 2.39(\mathrm{~s}, 3 \mathrm{H}), 1.92(\mathrm{~s}, 6 \mathrm{H}) ;{ }^{13} \mathrm{C} \mathrm{NMR}\left(\mathrm{CDCl}_{3}\right.$, $100 \mathrm{MHz}): \delta 143.1,142.8,139.0,136.0,133.9,130.8,129.1,123.2,122.1,120.1,109.9,20.9,17.2$.

\subsubsection{General Procedures for the Synthesis of Bis-Benzimidazolium Salts 3}

Procedure for bis-benzimidazolium salts $\mathbf{3 a - d}$. A $10 \mathrm{~mL}$ round-bottom flask was charged with dibromide $\mathbf{1 a}$ or $\mathbf{1 b}$ (1.0 equiv), benzimidazole $\mathbf{2 a}$ or $\mathbf{2 b}$ (2.0 equiv to bromide), and $\mathrm{MeCN}(5.0 \mathrm{~mL})$. After refluxing for $18 \mathrm{~h}$, the precipitate was formed. The precipitate was filtered off, washed with EtOAc, and dried to afford pure product.

Procedure for 3,3'-(1,3-Phenylenebis(methylene))bis(1-(4-methoxyphenyl)-1H-benzo[d] imidazol-3-ium) dibromide (3a). 1,3-bis-(bromomethyl)benzene 1a $(46.5 \mathrm{mg}, 0.18 \mathrm{mmol}$ ) and 1-(4-methoxyphenyl)-1 $H$-benzo[d]imidazole $2 \mathrm{a}(79.5 \mathrm{mg}, 0.36 \mathrm{mmol})$ were used to afford $3 \mathbf{a}$ as white solid in $88 \%$ yield $(0.10 \mathrm{~g})$. $\mathrm{Mp}=282-283^{\circ} \mathrm{C} ;{ }^{1} \mathrm{H}$ NMR (DMSO, $400 \mathrm{MHz}$ ): $\delta 10.36(\mathrm{~s}, 2 \mathrm{H}), 7.97(\mathrm{~s}, 1 \mathrm{H}), 7.94(\mathrm{~d}, J=8.0 \mathrm{~Hz}, 2 \mathrm{H}), 7.78-7.76(\mathrm{~m}, 6 \mathrm{H}), 7.68-7.58(\mathrm{~m}, 6 \mathrm{H})$, $7.48(\mathrm{t}, J=8.0 \mathrm{~Hz}, 1 \mathrm{H}), 7.26(\mathrm{~d}, J=8.8 \mathrm{~Hz}, 4 \mathrm{H}), 5.86(\mathrm{~s}, 4 \mathrm{H}), 3.89(\mathrm{~s}, 6 \mathrm{H}) ;{ }^{13} \mathrm{C}$ NMR (DMSO, $100 \mathrm{MHz}$ ): $\delta 158.6,140.8,132.4,129.7,128.7,127.6,127.0,126.7,125.4,124.9,124.8$, 123.7, 113.4, 112.1, 111.7, 57.7, 53.8. HRMS-MALDI-TOF $(m / z)[\mathrm{M}-\mathrm{Br}]^{+}$Calculated for $\mathrm{C}_{36} \mathrm{H}_{32} \mathrm{BrN}_{4} \mathrm{O}_{2}{ }^{+}:$631.1703, found: 631.1706 .

Procedure for 3,3'-(1,3-Phenylenebis(methylene))bis(1-mesityl-1H-benzo[d]imidazol-3-ium) dibromide (3b). 1,3-bis-(bromomethyl)benzene $1 \mathbf{a}(0.10 \mathrm{~g}, 0.38 \mathrm{mmol})$ and 1-(2,4,6-trimethylphenyl)-1 $1 \mathrm{H}$-benzimidazole $\mathbf{2 b}(0.18 \mathrm{~g}, 0.77 \mathrm{mmol})$ were used to afford $\mathbf{3 b}$ as white solid in $89 \%$ yield $(0.25 \mathrm{~g}) . \mathrm{Mp}=210-213{ }^{\circ} \mathrm{C} ;{ }^{1} \mathrm{H} \mathrm{NMR}\left(\mathrm{CDCl}_{3}, 400 \mathrm{MHz}\right): \delta 11.47(\mathrm{~s}, 2 \mathrm{H}), 8.51(\mathrm{~s}$, $1 \mathrm{H}), 8.23(\mathrm{~d}, J=8.4 \mathrm{~Hz}, 2 \mathrm{H}), 7.69(\mathrm{~d}, J=8.0 \mathrm{~Hz}, 2 \mathrm{H}), 7.67(\mathrm{t}, J=8.4 \mathrm{~Hz}, 2 \mathrm{H}), 7.54(\mathrm{t}, J=8.0 \mathrm{~Hz}$, $2 \mathrm{H}), 7.33(\mathrm{t}, J=8.0 \mathrm{~Hz}, 1 \mathrm{H}), 7.18(\mathrm{~d}, J=8.4 \mathrm{~Hz}, 2 \mathrm{H}), 7.06(\mathrm{~s}, 4 \mathrm{H}), 6.26(\mathrm{~s}, 4 \mathrm{H}), 2.37(\mathrm{~s}, 6 \mathrm{H})$, 1.99 (s, $12 \mathrm{H}) ;{ }^{13} \mathrm{C}$ NMR $\left(\mathrm{CDCl}_{3}, 100 \mathrm{MHz}\right): \delta 142.4,141.6,135.0,134.2,131.5,130.7,130.3$, $130.2,130.1,129.4,128.2,128.0,127.8,115.2,112.9,50.7,21.1,17.6$. HRMS-MALDI-TOF( $(m / z)$ [M-Br] ${ }^{+}$Calculated for $\mathrm{C}_{40} \mathrm{H}_{40} \mathrm{BrN}_{4}{ }^{+}$: 655.2431, found: 655.2435.

Procedure for 3,3'-(Pyridine-2,6-diylbis(methylene))bis(1-(4-methoxyphenyl)-1H-benzo[d] imidazol-3-ium) dibromide (3c). 2,6-bis-(bromomethyl)pyridine $1 \mathbf{b}(41.5 \mathrm{mg}, 0.16 \mathrm{mmol}$ ) 
and 1-(4-methoxyphenyl)-1H-benzo[d]imidazole $2 \mathrm{a}(72.1 \mathrm{mg}, 0.33 \mathrm{mmol})$ were used to afford $3 \mathrm{c}$ as white solid in $82 \%$ yield $(0.095 \mathrm{~g}) . \mathrm{Mp}=283-284{ }^{\circ} \mathrm{C} ;{ }^{1} \mathrm{H}$ NMR (DMSO, $400 \mathrm{MHz}): \delta 10.15(\mathrm{~s}, 2 \mathrm{H}), 8.06(\mathrm{t}, J=7.6 \mathrm{~Hz}, 1 \mathrm{H}), 7.79(\mathrm{t}, J=7.6 \mathrm{~Hz}, 4 \mathrm{H}), 7.68-7.55(\mathrm{~m}, 8 \mathrm{H})$, $7.47(\mathrm{t}, J=7.6 \mathrm{~Hz}, 2 \mathrm{H}), 7.20(\mathrm{~d}, J=8.4 \mathrm{~Hz}, 4 \mathrm{H}), 5.92(\mathrm{~s}, 4 \mathrm{H}), 3.89(\mathrm{~s}, 6 \mathrm{H}) ;{ }^{13} \mathrm{C}$ NMR (DMSO, $100 \mathrm{MHz}): \delta 160.5,152.9,144.8,142.8,131.0,130.97,127.2,126.8,126.5,125.4,123.0,115.3$, $113.8,113.4,55.7,50.8$. HRMS-MALDI-TOF $(m / z)[\mathrm{M}-\mathrm{Br}]^{+}$Calculated for $\mathrm{C}_{35} \mathrm{H}_{31} \mathrm{BrN}_{5} \mathrm{O}_{2}{ }^{+}$: 632.1656, found: 632.1657.

Procedure for 3,3'-(Pyridine-2,6-diylbis(methylene))bis(1-mesityl-1H-benzo[d]imidazol-3ium) dibromide (3d). 2,6-bis-(bromomethyl)pyridine $\mathbf{1 b}(24.7 \mathrm{mg}, 0.09 \mathrm{mmol})$ and 1-(2,4,6trimethylphenyl)- $1 H$-benzimidazole $\mathbf{2 b}(44.4 \mathrm{mg}, 0.18 \mathrm{mmol})$ were used to afford $\mathbf{3 d}$ as white solid in 50\% yield $(0.034 \mathrm{~g}) . \mathrm{Mp}=204-205{ }^{\circ} \mathrm{C} ;{ }^{1} \mathrm{H} \mathrm{NMR}\left(\mathrm{CDCl}_{3}, 400 \mathrm{MHz}\right): \delta 11.57(\mathrm{~s}$, $2 \mathrm{H}), 7.97(\mathrm{~d}, J=8.0 \mathrm{~Hz}, 2 \mathrm{H}), 7.78-7.75(\mathrm{~m}, 5 \mathrm{H}), 7.58(\mathrm{t}, J=8.0 \mathrm{~Hz}, 2 \mathrm{H}), 7.23(\mathrm{~d}, J=8.0 \mathrm{~Hz}$, 2H), $7.10(\mathrm{~s}, 4 \mathrm{H}), 6.42(\mathrm{~s}, 4 \mathrm{H}), 2.40(\mathrm{~s}, 6 \mathrm{H}), 2.04(\mathrm{~s}, 12 \mathrm{H}) ;{ }^{13} \mathrm{C} \mathrm{NMR}\left(\mathrm{CDCl}_{3}, 400 \mathrm{MHz}\right): \delta$ 152.9, 143.1, 141.8, 139.5, 135.1, 131.5, 130.9, 130.2, 128.6, 128.1, 127.9, 123.5, 114.6, 113.0, 52.2, 21.2, 17.7; HRMS-MALDI-TOF $(m / z)$ [M-Br] ${ }^{+}$Calculated for $\mathrm{C}_{39} \mathrm{H}_{39} \mathrm{BrN}_{5}{ }^{+}: 656.2383$, found: 656.2377 .

General Procedures for Preparation of bis-benzimidazolium salts $3 \mathbf{e}$ and $3 \mathbf{f}$. A $10 \mathrm{~mL}$ round-bottom flask was charged with dibromide 1c (1.0 equiv), benzimidazole $\mathbf{2 a}$ or $\mathbf{2 b}$ (2.0 equiv to bromide), and $\mathrm{MeCN}(5.0 \mathrm{~mL})$. After refluxing for $18 \mathrm{~h}$, the precipitate was formed. The precipitate was filtered off, washed with EtOAc, and dried to afford pure product.

Procedure for 3,3'-([1,1'-Biphenyl]-2,2' -diylbis(methylene))bis(1-(4-methoxyphenyl)-1Hbenzo[d]imidazol-3-ium) dibromide (3e). 2,2'-bis((bromomethyl)benzene 1c (46.0 mg, $0.14 \mathrm{mmol}$ ) and 1-(4-methoxyphenyl)- $1 H$-benzo[d]imidazole $2 \mathrm{a}(62.2 \mathrm{mg}, 0.29 \mathrm{mmol})$ were used to afford $3 \mathbf{e}$ as white solid in $78 \%$ yield $(0.083 \mathrm{~g}) . \mathrm{Mp}=200-201{ }^{\circ} \mathrm{C} ;{ }^{1} \mathrm{H} \mathrm{NMR}\left(\mathrm{CDCl}_{3}\right.$, $400 \mathrm{MHz}): \delta 10.52(\mathrm{~s}, 2 \mathrm{H}), 7.68-7.53(\mathrm{~m}, 12 \mathrm{H}), 7.49(\mathrm{~d}, J=7.6 \mathrm{~Hz}, 2 \mathrm{H}), 7.35(\mathrm{t}, J=7.6 \mathrm{~Hz}, 2 \mathrm{H})$, $7.26(\mathrm{t}, J=7.6 \mathrm{~Hz}, 2 \mathrm{H}), 7.11(\mathrm{~d}, J=8.8 \mathrm{~Hz}, 4 \mathrm{H}), 6.88(\mathrm{~d}, J=7.6 \mathrm{~Hz}, 2 \mathrm{H}), 6.26(\mathrm{~d}, J=16.0 \mathrm{~Hz}$, 2H), $5.91(\mathrm{~d}, J=16.0 \mathrm{~Hz}, 2 \mathrm{H}), 3.87(\mathrm{~s}, 6 \mathrm{H}) ;{ }^{13} \mathrm{C} \mathrm{NMR}\left(\mathrm{CDCl}_{3}, 100 \mathrm{MHz}\right): \delta 161.0,142.0,138.5$, 131.2, 131.1, 131.0, 129.8, 129.7, 129.4, 129.3, 127.72, 127.65, 126.1, 125.1, 115.7, 114.2, 113.5, 55.8, 50.3. HRMS-MALDI-TOF $(m / z)[\mathrm{M}-\mathrm{Br}]^{+}$Calculated for $\mathrm{C}_{42} \mathrm{H}_{36} \mathrm{BrN}_{4} \mathrm{O}_{2}{ }^{+}:$707.2016, found: 707.2017.

Procedure for 3,3'-([1,1'-Biphenyl]-2,2'-diylbis(methylene))bis(1-mesityl-1H-benzo[d] imidazol-3-ium) dibromide (3f). 2,2'-bis((bromomethyl)benzene 1c (36.5 mg, $0.11 \mathrm{mmol})$ and 1-(2,4,6-trimethylphenyl)-1H-benzimidazole $2 \mathbf{b}(54.3 \mathrm{mg}, 0.24 \mathrm{mmol})$ were used to afford $3 \mathrm{f}$ as white solid in $68 \%$ yield $(0.059 \mathrm{~g}) . \mathrm{Mp}=297-298{ }^{\circ} \mathrm{C} ;{ }^{1} \mathrm{H} \mathrm{NMR}\left(\mathrm{CDCl}_{3}, 400 \mathrm{MHz}\right)$ : $\delta 11.00(\mathrm{~s}, 2 \mathrm{H}), 7.89(\mathrm{~d}, J=7.6 \mathrm{~Hz}, 2 \mathrm{H}), 7.70(\mathrm{t}, J=7.6 \mathrm{~Hz}, 2 \mathrm{H}), 7.61(\mathrm{t}, J=7.6 \mathrm{~Hz}, 2 \mathrm{H}), 7.52$ $(\mathrm{d}, J=7.6 \mathrm{~Hz}, 2 \mathrm{H}), 7.46(\mathrm{t}, J=7.6 \mathrm{~Hz}, 2 \mathrm{H}), 7.37(\mathrm{t}, J=7.6 \mathrm{~Hz}, 2 \mathrm{H}), 7.25(\mathrm{~d}, J=7.6 \mathrm{~Hz}, 2 \mathrm{H})$, $7.07(\mathrm{~s}, 4 \mathrm{H}), 7.04(\mathrm{~d}, J=7.6 \mathrm{~Hz}, 2 \mathrm{H}), 6.55(\mathrm{~d}, J=16.8 \mathrm{~Hz}, 2 \mathrm{H}), 5.97(\mathrm{~d}, J=16.8 \mathrm{~Hz}, 2 \mathrm{H}), 2.38$ (s, 6H), 2.05 (s, 6H), $2.01(\mathrm{~s}, 6 \mathrm{H}) ;{ }^{13} \mathrm{C} \mathrm{NMR}\left(\mathrm{CDCl}_{3}, 100 \mathrm{MHz}\right): \delta 143.4,141.7,138.0,135.1$, 135.0, 131.5, 131.4, 131.3, 130.6, 130.2, 129.44, 129.39, 128.3, 128.1, 126.7, 114.7, 113.0, 49.7, 21.2, 17.8, 17.7. HRMS-MALDI-TOF $(m / z)[\mathrm{M}-\mathrm{Br}]^{+}$Calculated for $\mathrm{C}_{46} \mathrm{H}_{44} \mathrm{BrN}_{4}{ }^{+}:$731.2744, found: 731.2742 .

\subsubsection{General Procedures for Suzuki-Miyaura Cross-Coupling Reactions under $\mathrm{N}_{2}$}

All manipulations were carried out under nitrogen using dried solvent. $\mathrm{Pd}(\mathrm{OAc})_{2}$ $(1 \mathrm{~mol} \%)$, salt $3 \mathbf{b}(1 \mathrm{~mol} \%)$ and 1,4-dioxane $(3 \mathrm{~mL})$ were charged to the Schlenk tube at $80{ }^{\circ} \mathrm{C}$ for $1 \mathrm{~h}$, followed by the addition of arylboronic acid 5 (1.50 mmol), aryl chloride 4 $(1.00 \mathrm{mmol})$, and $\mathrm{K}_{3} \mathrm{PO}_{4} \cdot \mathrm{H}_{2} \mathrm{O}(3.00 \mathrm{mmol})$ at the prescribed temperature for the prescribed time. After completion of the reaction, monitored by TLC, the reaction was quenched by water $(3.0 \mathrm{~mL})$. The aqueous layer was extracted with EtOAc $(3.0 \mathrm{~mL} \times 3)$. The organic layer was dried over anhydrous $\mathrm{MgSO}_{4}$ and then filtered. The solvent was evaporated under reduced pressure and the corresponding crude product of the Suzuki-Miyaura coupling reaction was purified by chromatography. 
4-Acetylbiphenyl (6aa) [29]. ${ }^{1} \mathrm{H}$ NMR ( $\left.\mathrm{CDCl}_{3}, 400 \mathrm{MHz}\right): \delta 8.04$ (d, J = 7.6 Hz, 2H), 7.69 (d, $J=7.6 \mathrm{~Hz}, 2 \mathrm{H}), 7.63(\mathrm{~d}, J=7.6 \mathrm{~Hz}, 2 \mathrm{H}), 7.47(\mathrm{t}, J=7.6 \mathrm{~Hz}, 2 \mathrm{H}), 7.43-7.38(\mathrm{~m}, 1 \mathrm{H}), 2.64(\mathrm{~s}$, $3 \mathrm{H}) ;{ }^{13} \mathrm{C} \mathrm{NMR}\left(\mathrm{CDCl}_{3}, 100 \mathrm{MHz}\right): \delta 197.7,145.7,139.8,135.8,128.92,128.88,128.2,127.2$, 127.2, 26.6.

3-Acetylbiphenyl (6ba) [35]. ${ }^{1} \mathrm{H}$ NMR ( $\left.\mathrm{CDCl}_{3}, 400 \mathrm{MHz}\right): \delta 8.19$ (s, $\left.1 \mathrm{H}\right), 7.94(\mathrm{~d}, J=7.6 \mathrm{~Hz}$, $1 \mathrm{H}), 7.80(\mathrm{~d}, J=7.6 \mathrm{~Hz}, 1 \mathrm{H}), 7.63(\mathrm{~d}, J=7.2 \mathrm{~Hz}, 2 \mathrm{H}), 7.54(\mathrm{t}, J=7.6 \mathrm{~Hz}, 1 \mathrm{H}), 7.47(\mathrm{t}, J=7.2 \mathrm{~Hz}$, 2H), 7.41-7.37 (m, 1H), $2.66(\mathrm{~s}, 3 \mathrm{H}) ;{ }^{13} \mathrm{C} \mathrm{NMR}\left(\mathrm{CDCl}_{3}, 100 \mathrm{MHz}\right): \delta 197.8,141.4,139.9,137.4$, $131.5,128.8,128.7,127.6,127.0,126.95,126.7,26.5$.

2-Acetylbiphenyl (6ca) [36]. ${ }^{1} \mathrm{H}$ NMR $\left(\mathrm{CDCl}_{3}, 400 \mathrm{MHz}\right): \delta 7.57-7.50(\mathrm{~m}, 2 \mathrm{H}), 7.44-7.39(\mathrm{~m}$, $5 \mathrm{H}), 7.36-7.34(\mathrm{~m}, 2 \mathrm{H}), 2.01(\mathrm{~s}, 3 \mathrm{H}) ;{ }^{13} \mathrm{C} \mathrm{NMR}\left(\mathrm{CDCl}_{3}, 100 \mathrm{MHz}\right): \delta 205.0,140.8,140.6,140.5$, $130.7,130.2,128.8,128.6,127.85,127.82,127.4,30.4$.

4'-Phenylpropiophenone (6da) [29]. ${ }^{1} \mathrm{H}$ NMR ( $\left.\mathrm{CDCl}_{3}, 400 \mathrm{MHz}\right): \delta 8.05(\mathrm{~d}, J=8.4 \mathrm{~Hz}, 2 \mathrm{H})$, $7.68(\mathrm{~d}, J=8.4 \mathrm{~Hz}, 2 \mathrm{H}), 7.63(\mathrm{~d}, J=7.8 \mathrm{~Hz}, 2 \mathrm{H}), 7.47(\mathrm{t}, J=7.8 \mathrm{~Hz}, 2 \mathrm{H}), 7.43-7.38(\mathrm{~m}, 1 \mathrm{H})$, $3.05(\mathrm{q}, J=7.2 \mathrm{~Hz}, 2 \mathrm{H}), 1.26(\mathrm{t}, J=7.2 \mathrm{~Hz}, 3 \mathrm{H}) ;{ }^{13} \mathrm{C} \mathrm{NMR}\left(\mathrm{CDCl}_{3}, 100 \mathrm{MHz}\right): \delta 200.5,145.5$, $139.9,135.6,128.9,128.6,128.2,127.23,127.19,31.8,8.3$.

3'-Phenylpropiophenone (6ea) [29]. ${ }^{1} \mathrm{H}$ NMR $\left(\mathrm{CDCl}_{3}, 400 \mathrm{MHz}\right): \delta 8.20(\mathrm{~s}, 1 \mathrm{H}), 7.95(\mathrm{~d}$, $J=7.6 \mathrm{~Hz}, 1 \mathrm{H}), 7.78(\mathrm{~d}, J=7.6 \mathrm{~Hz}, 1 \mathrm{H}), 7.63(\mathrm{~d}, J=7.6 \mathrm{~Hz}, 2 \mathrm{H}), 7.53(\mathrm{t}, J=7.6 \mathrm{~Hz}, 1 \mathrm{H}), 7.48$ $(\mathrm{t}, J=7.6 \mathrm{~Hz}, 2 \mathrm{H}), 7.43-7.36(\mathrm{~m}, 1 \mathrm{H}), 3.07(\mathrm{q}, J=7.2 \mathrm{~Hz}, 2 \mathrm{H}), 1.27(\mathrm{t}, J=7.2 \mathrm{~Hz}, 3 \mathrm{H}) ;{ }^{13} \mathrm{C}$ $\operatorname{NMR}\left(\mathrm{CDCl}_{3}, 100 \mathrm{MHz}\right): \delta 200.7,141.6,140.2,137.3,131.5,129.0,128.9,127.7,127.1,126.8$, $126.6,31.9,8.2$.

4-Nitrobiphenyl (6fa) [37]. ${ }^{1} \mathrm{H}$ NMR $\left(\mathrm{CDCl}_{3}, 400 \mathrm{MHz}\right): \delta 8.30(\mathrm{~d}, J=8.4 \mathrm{~Hz}, 2 \mathrm{H}), 7.74(\mathrm{~d}$, $J=8.4 \mathrm{~Hz}, 2 \mathrm{H}), 7.63(\mathrm{~d}, J=7.2 \mathrm{~Hz}, 2 \mathrm{H}), 7.55-7.41(\mathrm{~m}, 3 \mathrm{H}) ;{ }^{13} \mathrm{C} \mathrm{NMR}\left(\mathrm{CDCl}_{3}, 100 \mathrm{MHz}\right): \delta$ 147.6, 147.0, 138.7, 129.1, 128.9, 127.8, 127.3, 124.1.

2-Nitrobiphenyl (6ga) [37]. ${ }^{1} \mathrm{H}$ NMR ( $\left.\mathrm{CDCl}_{3}, 400 \mathrm{MHz}\right): 87.86(\mathrm{~d}, J=7.6 \mathrm{~Hz}, 1 \mathrm{H}), 7.62(\mathrm{t}$, $J=7.6 \mathrm{~Hz}, 1 \mathrm{H}), 7.51-7.39(\mathrm{~m}, 5 \mathrm{H}), 7.34-7.32(\mathrm{~m}, 2 \mathrm{H}) ;{ }^{13} \mathrm{C} \mathrm{NMR}\left(\mathrm{CDCl}_{3}, 100 \mathrm{MHz}\right): \delta 149.2$, 137.3, 136.3, 132.2, 131.9, 128.7, 128.2, 128.1, 127.9, 124.0.

2,4-Dinitrobiphenyl (6ha) [29]. ${ }^{1} \mathrm{H}$ NMR $\left(\mathrm{CDCl}_{3}, 400 \mathrm{MHz}\right): \delta 8.71(\mathrm{~d}, J=2.4 \mathrm{~Hz}, 1 \mathrm{H}), 8.47$ $(\mathrm{dd}, J=8.8,2.4 \mathrm{~Hz}, 1 \mathrm{H}), 7.67(\mathrm{~d}, J=8.8 \mathrm{~Hz}, 1 \mathrm{H}), 7.50-7.47(\mathrm{~m}, 3 \mathrm{H}), 7.36-7.33(\mathrm{~m}, 2 \mathrm{H}) ;{ }^{13} \mathrm{C}$ $\operatorname{NMR}\left(\mathrm{CDCl}_{3}, 100 \mathrm{MHz}\right): \delta$ 148.8, 146.6, 142.1, 135.1, 133.2, 129.4, 128.9, 127.5, 126.4, 119.6. 4-Cyanobiphenyl (6ia) [38]. ${ }^{1} \mathrm{H}$ NMR $\left(\mathrm{CDCl}_{3}, 400 \mathrm{MHz}\right): \delta 7.73(\mathrm{~d}, J=8.4 \mathrm{~Hz}, 2 \mathrm{H}), 7.69(\mathrm{~d}$, $J=8.4 \mathrm{~Hz}, 2 \mathrm{H}), 7.59(\mathrm{~d}, J=7.6 \mathrm{~Hz}, 2 \mathrm{H}), 7.49(\mathrm{t}, J=7.6 \mathrm{~Hz}, 2 \mathrm{H}), 7.46-7.40(\mathrm{~m}, 1 \mathrm{H}) ;{ }^{13} \mathrm{C} \mathrm{NMR}$ $\left(\mathrm{CDCl}_{3}, 100 \mathrm{MHz}\right): \delta 145.2,138.7,132.2,128.8,128.4,127.3,126.9,118.6,110.5$.

1-(4-(Naphthalen-1-yl)phenyl)ethan-1-one (6ab) [29]. ${ }^{1} \mathrm{H}$ NMR $\left(\mathrm{CDCl}_{3}, 400 \mathrm{MHz}\right): \delta 8.10$ $(\mathrm{d}, J=7.6 \mathrm{~Hz}, 2 \mathrm{H}), 7.94-7.84(\mathrm{~m}, 3 \mathrm{H}), 7.61(\mathrm{~d}, J=7.6 \mathrm{~Hz}, 2 \mathrm{H}), 7.57-7.43(\mathrm{~m}, 4 \mathrm{H}), 2.69(\mathrm{~s}, 3 \mathrm{H})$; ${ }^{13} \mathrm{C} \mathrm{NMR}\left(\mathrm{CDCl}_{3}, 100 \mathrm{MHz}\right)$ : $\delta 197.9,145.8,139.0,135.9,133.7,131.1,130.3,128.4,128.35$, $128.33,126.9,126.4,126.0,125.5,125.3,26.7$.

1-(4-(Naphthalen-1-yl)phenyl)propan-1-one (6db) [29]. ${ }^{1} \mathrm{H}$ NMR ( $\left.\mathrm{CDCl}_{3}, 400 \mathrm{MHz}\right): \delta 8.10$ $(\mathrm{d}, J=8.0 \mathrm{~Hz}, 2 \mathrm{H}), 7.94-7.84(\mathrm{~m}, 3 \mathrm{H}), 7.60(\mathrm{~d}, J=8.0 \mathrm{~Hz}, 2 \mathrm{H}), 7.57-7.42(\mathrm{~m}, 4 \mathrm{H}), 3.09(\mathrm{q}$, $J=7.2 \mathrm{~Hz}, 2 \mathrm{H}), 1.29(\mathrm{t}, J=7.2 \mathrm{~Hz}, 3 \mathrm{H}) ;{ }^{13} \mathrm{C} \mathrm{NMR}\left(\mathrm{CDCl}_{3}, 100 \mathrm{MHz}\right): \delta 200.1,145.2,138.8$, 135.5, 133.6, 130.9, 130.0, 128.2, 128.1, 127.8, 126.7, 126.2, 125.8, 125.3, 125.1, 31.6, 8.1.

1-(3-(Naphthalen-1-yl)phenyl)propan-1-one (6eb) [29]. ${ }^{1} \mathrm{H}$ NMR ( $\left.\mathrm{CDCl}_{3}, 400 \mathrm{MHz}\right): \delta 8.09$ $(\mathrm{s}, 1 \mathrm{H}), 8.04(\mathrm{~d}, J=8.4 \mathrm{~Hz}, 1 \mathrm{H}), 7.94-7.89(\mathrm{~m}, 2 \mathrm{H}), 7.81(\mathrm{~d}, J=8.0 \mathrm{~Hz}, 1 \mathrm{H}), 7.69(\mathrm{~d}, J=8.0 \mathrm{~Hz}$, $1 \mathrm{H}), 7.61-7.50(\mathrm{~m}, 3 \mathrm{H}), 7.44(\mathrm{t}, J=8.4 \mathrm{~Hz}, 2 \mathrm{H}), 3.05(\mathrm{q}, J=7.2 \mathrm{~Hz}, 2 \mathrm{H}), 1.25(\mathrm{t}, J=7.2 \mathrm{~Hz}, 3 \mathrm{H})$; ${ }^{13} \mathrm{C}$ NMR $\left(\mathrm{CDCl}_{3}, 100 \mathrm{MHz}\right): \delta 200.1,140.7,138.8,136.7,134.0,133.5,131.1,129.2,128.2$, $128.1,127.8,126.7,126.5,126.0,125.6,125.2,125.0,31.5,7.9$.

1-(4-Nitrophenyl)naphthalene (6fb) [37]. ${ }^{1} \mathrm{H}$ NMR $\left(\mathrm{CDCl}_{3}, 400 \mathrm{MHz}\right): \delta 8.37(\mathrm{~d}, J=8.4 \mathrm{~Hz}$, $2 \mathrm{H}), 7.94(\mathrm{dd}, J=8.4,2.4 \mathrm{~Hz}, 2 \mathrm{H}), 7.78(\mathrm{~d}, J=8.4 \mathrm{~Hz}, 1 \mathrm{H}), 7.68(\mathrm{~d}, J=8.4 \mathrm{~Hz}, 2 \mathrm{H}), 7.59-7.41$ $(\mathrm{m}, 4 \mathrm{H}) ;{ }^{13} \mathrm{C} \mathrm{NMR}\left(\mathrm{CDCl}_{3}, 100 \mathrm{MHz}\right): \delta 147.6,147.1,137.7,133.7,130.9,128.9,128.5,127.1$, $126.7,126.2,125.3,125.1,123.6$.

4-(1-Naphthyl)benzonitrile (6ib) [38]. ${ }^{1} \mathrm{H}$ NMR $\left(\mathrm{CDCl}_{3}, 400 \mathrm{MHz}\right): 87.93(\mathrm{~d}, J=7.2 \mathrm{~Hz}$, $2 \mathrm{H}), 7.80-7.76(\mathrm{~m}, 3 \mathrm{H}), 7.62(\mathrm{~d}, J=8.4 \mathrm{~Hz}, 2 \mathrm{H}), 7.57-7.45(\mathrm{~m}, 3 \mathrm{H}), 7.40(\mathrm{~d}, J=7.2 \mathrm{~Hz}, 1 \mathrm{H})$; 
${ }^{13} \mathrm{C}$ NMR $\left(\mathrm{CDCl}_{3}, 100 \mathrm{MHz}\right): \delta 145.2,137.8,133.5,131.8,130.6,130.4,128.5,128.3,126.8$, $126.4,125.9,125.1,124.9,118.6,110.8$.

Diphenylmethane (6ja) [39]. ${ }^{1} \mathrm{H}$ NMR $\left(\mathrm{CDCl}_{3}, 400 \mathrm{MHz}\right): 87.31-7.27(\mathrm{~m}, 4 \mathrm{H}), 7.22-7.20$ (m, 6H), 3.99 (s, 2H); ${ }^{13} \mathrm{C} \mathrm{NMR}\left(\mathrm{CDCl}_{3}, 100 \mathrm{MHz}\right): \delta 141.1,128.9,128.4,126.0,41.9$.

1-Benzylnapthalene (6jb) [40]. ${ }^{1} \mathrm{H}$ NMR $\left(\mathrm{CDCl}_{3}, 400 \mathrm{MHz}\right): \delta 7.99(\mathrm{~d}, J=8.0 \mathrm{~Hz}, 1 \mathrm{H})$, 7.86-7.84 (m, 1H), 7.76 (d, $J=8.0 \mathrm{~Hz}, 1 \mathrm{H}), 7.46-7.40(\mathrm{~m}, 3 \mathrm{H}), 7.29-7.24(\mathrm{~m}, 3 \mathrm{H}), 7.20-7.16$ $(\mathrm{m}, 3 \mathrm{H}), 4.45(\mathrm{~s}, 2 \mathrm{H}) ;{ }^{13} \mathrm{C} \mathrm{NMR}\left(\mathrm{CDCl}_{3}, 100 \mathrm{MHz}\right): \delta 140.5,136.5,133.9,132.1,128.6,128.58$, $128.4,127.2,127.1,125.9,125.9,125.5,124.2,38.9$.

4-Methylbiphenyl (6ka) [37]. ${ }^{1} \mathrm{H}$ NMR ( $\left.\mathrm{CDCl}_{3}, 400 \mathrm{MHz}\right): 87.58(\mathrm{~d}, J=7.2 \mathrm{~Hz}, 2 \mathrm{H}), 7.49(\mathrm{~d}$, $J=8.0 \mathrm{~Hz}, 2 \mathrm{H}), 7.42(\mathrm{t}, J=7.2 \mathrm{~Hz}, 2 \mathrm{H}), 7.34-7.30(\mathrm{~m}, 1 \mathrm{H}), 7.25(\mathrm{~d}, J=8.0 \mathrm{~Hz}, 2 \mathrm{H}), 2.40(\mathrm{~s}$, $3 \mathrm{H}) ;{ }^{13} \mathrm{C} \mathrm{NMR}\left(\mathrm{CDCl}_{3}, 100 \mathrm{MHz}\right): \delta 141.1,138.3,137.0,129.5,128.7,127.0,21.1$.

3-Methylbiphenyl (6la) [41]. ${ }^{1} \mathrm{H}$ NMR ( $\left.\mathrm{CDCl}_{3}, 400 \mathrm{MHz}\right): \delta 7.60(\mathrm{~d}, J=7.2 \mathrm{~Hz}, 2 \mathrm{H}), 7.46-$ $7.40(\mathrm{~m}, 4 \mathrm{H}), 7.37-7.33(\mathrm{~m}, 2 \mathrm{H}), 7.18(\mathrm{~d}, J=7.2 \mathrm{~Hz}, 1 \mathrm{H}), 2.44(\mathrm{~s}, 3 \mathrm{H}) ;{ }^{13} \mathrm{C} \mathrm{NMR}\left(\mathrm{CDCl}_{3}\right.$, $100 \mathrm{MHz}): \delta 141.3,141.2,138.2,128.6,127.9,127.87,127.1,124.2,21.4$.

2-Methylbiphenyl (6ma) [29]. ${ }^{1} \mathrm{H}$ NMR ( $\left.\mathrm{CDCl}_{3}, 400 \mathrm{MHz}\right): \delta 7.41$ (t, $\left.J=7.2 \mathrm{~Hz}, 2 \mathrm{H}\right), 7.35$ $7.31(\mathrm{~m}, 3 \mathrm{H}), 7.26-7.24(\mathrm{~m}, 4 \mathrm{H}),, 2.27(\mathrm{~s}, 3 \mathrm{H}) ;{ }^{13} \mathrm{C} \mathrm{NMR}\left(\mathrm{CDCl}_{3}, 100 \mathrm{MHz}\right): \delta 141.9,141.89$, 135.3, 130.3, 129.8, 129.1, 128.0, 127.2, 126.7, 125.7, 20.4 .

1-(2,4-Dinitrophenyl)naphthalene (6hb) [42]. ${ }^{1} \mathrm{H}$ NMR ( $\left.\mathrm{CDCl}_{3}, 400 \mathrm{MHz}\right): \delta 8.93(\mathrm{~s}, 1 \mathrm{H})$, $8.55(\mathrm{~d}, J=8.4 \mathrm{~Hz}, 1 \mathrm{H}), 7.96(\mathrm{t}, J=9.2 \mathrm{~Hz}, 2 \mathrm{H}), 7.74(\mathrm{~d}, J=8.4 \mathrm{~Hz}, 1 \mathrm{H}), 7.57-7.52(\mathrm{~m}, 2 \mathrm{H})$, $7.46(\mathrm{t}, J=7.6 \mathrm{~Hz}, 1 \mathrm{H}), 7.38-7.34(\mathrm{~m}, 2 \mathrm{H}) ;{ }^{13} \mathrm{C} \mathrm{NMR}\left(\mathrm{CDCl}_{3}, 100 \mathrm{MHz}\right): \delta 149.8,147.4,141.6$, $134.5,133.4,133.2,130.7,129.7,128.8,127.2,126.6,126.5,126.1,125.2,124.1,119.9$.

1-(2-Nitrophenyl)naphthalene (6gb) [43]. ${ }^{1} \mathrm{H}$ NMR $\left(\mathrm{CDCl}_{3}, 400 \mathrm{MHz}\right): \delta 8.08(\mathrm{~d}, J=8.4 \mathrm{~Hz}$, $1 \mathrm{H}), 7.91(\mathrm{~d}, J=8.4 \mathrm{~Hz}, 2 \mathrm{H}), 7.71(\mathrm{t}, J=7.2 \mathrm{~Hz}, 1 \mathrm{H}), 7.61(\mathrm{t}, J=8.4 \mathrm{~Hz}, 1 \mathrm{H}), 7.52-7.39(\mathrm{~m}$, $5 \mathrm{H}), 7.35(\mathrm{~d}, J=7.2 \mathrm{~Hz}, 1 \mathrm{H}) ;{ }^{13} \mathrm{C} \mathrm{NMR}\left(\mathrm{CDCl}_{3}, 100 \mathrm{MHz}\right): \delta 149.8,135.5,135.3,133.4,133.1$, $132.5,131.4,128.6,128.6,128.5,126.6,126.0,125.6,125.2,124.8,124.23$.

\section{Conclusions}

In summary, a series of new xylyl-linked bis-benzimidazolium salts $\mathbf{3 a}-\mathbf{3 f}$ were effectively synthesized via a simple synthetic route using cheap reactants. The changing of both the linkers and wingtips of the xylyl-linked bis-benzimidazolium salts $\mathbf{3 a}-\mathbf{3 f}$ led to different steric and electronic effects being observed. It was found in the Suzuki-Miyaura cross-coupling reactions of aryl chlorides that bis-benzimidazolium salts featuring benzene as a xylyl-linked spacer ( $\mathbf{3 a}$ and $\mathbf{3 b}$ ) led to higher yields of the respective products being formed, whereas when pyridine ( $\mathbf{3} \mathbf{c}$ and $\mathbf{3 d}$ ) and biphenyl (3e and $\mathbf{3 f}$ ) were used as linkers lower yields were observed. Comparing the bis-NHC precursors $3 \mathbf{a}$ and $\mathbf{3 b}$, the precursor with a sterically demanding wingtip (3b) led to the product being formed in a higher yield than when a precursor was used with an electronically demanding wingtip (3a). Various aryl chlorides, including carbonyl-, nitro-, and nitrile-functionalized compounds, were coupled with arylboronic acids to give the respective products in good to excellent yields at $80-100{ }^{\circ} \mathrm{C}$ using the in-situ-generated bis-NHC $3 \mathbf{b} / \mathrm{Pd}$ catalyst system, with a loading in the range of $0.5-2.0 \mathrm{~mol} \%$. In addition, in di-ortho-substituted biaryl syntheses excellent yields were observed when 1-chloro-2,4-dinitrobenzene and 1-chloro-2-nitrobenzene were coupled with 1-naphthylboronic acid.

Supplementary Materials: The following are available online at https: / www.mdpi.com/article/10 $.3390 /$ catal11070817/s1, Figure S1: ${ }^{1} \mathrm{H}$ NMR spectrum of 2-(2' $4^{\prime}, 6^{\prime}$-trimethylanilido)nitrobenzene S1 in $\mathrm{CDCl}_{3}$, Figure S2: ${ }^{13} \mathrm{C}$ NMR spectrum of 2-(2', $4^{\prime}, 6^{\prime}$-trimethylanilido)nitrobenzene S1 in $\mathrm{CDCl}_{3}$, Figure S3: ${ }^{1} \mathrm{H}$ NMR spectrum of $N-\left(2^{\prime}, 4^{\prime}, 6^{\prime}\right.$-trimethylphenyl)-1,2-phenylenediamine S1 in $\mathrm{CDCl}_{3}$, Figure S4: ${ }^{13} \mathrm{C}$ NMR spectrum of $N-\left(2^{\prime}, 4^{\prime}, 6^{\prime}\right.$-trimethylphenyl)-1,2-phenylenediamine S2 in $\mathrm{CDCl}_{3}$, Figure S5: ${ }^{1} \mathrm{H}$ NMR spectrum of 1-(2,4,6-trimethylphenyl)-1H-benzimidazole $\mathbf{2 b}$ in $\mathrm{CDCl}_{3}$, Figure $\mathrm{S} 6:{ }^{13} \mathrm{C}$ NMR spectrum of 1-(2,4,6-trimethylphenyl)- $1 \mathrm{H}$-benzimidazole $\mathbf{2 b}$ in $\mathrm{CDCl}_{3}$, Figure S7: ${ }^{1} \mathrm{H}$ NMR spectrum of (3,3'-(1,3-phenylenebis(methylene))bis(1-(4-methoxyphenyl)- $1 \mathrm{H}^{-}$ benzo[d]imidazol-3-ium)) dibromide (3a) in DMSO- $\mathrm{d}_{6}$, Figure S8: ${ }^{13} \mathrm{C}$ NMR spectrum of $\left(3,3^{\prime}{ }^{\prime}\right.$ 
(1,3-phenylenebis(methylene))bis(1-(4-methoxyphenyl)-1H-benzo[d]imidazol-3-ium)) dibromide (3a) in DMSO- $\mathrm{d}_{6}$, Figure S9: ${ }^{1} \mathrm{H}$ NMR spectrum of (3,3'-(1,3-phenylenebis(methylene))bis(1-mesityl$1 \mathrm{H}$-benzo[d]imidazol-3-ium)) dibromide (3b) in $\mathrm{CDCl}_{3}$, Figure S10: ${ }^{13} \mathrm{C}$ NMR spectrum of $\left(3,3^{\prime}\right.$ (1,3-phenylenebis(methylene))bis(1-mesityl-1H-benzo[d]imidazol-3-ium)) dibromide (3b) in $\mathrm{CDCl}_{3}$, Figure S11: ${ }^{1} \mathrm{H}$ NMR spectrum of (3,3'-(pyridine-2,6-diylbis(methylene))bis(1-(4-methoxyphenyl)$1 H$-benzo[d]imidazol-3-ium)) dibromide (3c) in DMSO-d 6 , Figure S12: ${ }^{13} \mathrm{C}$ NMR spectrum of $\left(3,3^{\prime}\right.$ (pyridine-2,6-diylbis(methylene))bis(1-(4-methoxyphenyl)-1H-benzo[d]imidazol-3-ium)) dibromide (3c) in DMSO- $\mathrm{d}_{6}$, Figure S13: ${ }^{1} \mathrm{H}$ NMR spectrum of $\left(3,3^{\prime}\right.$-(pyridine-2,6-diylbis(methylene))bis(1mesityl-1H-benzo[d] imidazol-3-ium)) dibromide (3d) in $\mathrm{CDCl}_{3}$, Figure S14: ${ }^{13} \mathrm{C}$ NMR spectrum of (3,3'-(pyridine-2,6-diylbis(methylene))bis(1-mesityl-1H-benzo[d]imidazol-3-ium)) dibromide (3d) in $\mathrm{CDCl}_{3}$, Figure S15: ${ }^{1} \mathrm{H}$ NMR spectrum of 3,3'-([1,1'-biphenyl]-2,2'-diylbis(methylene))bis(1-(4methoxyphenyl)- $1 \mathrm{H}$-benzo[d]imidazol-3-ium) dibromide (3e) in $\mathrm{CDCl}_{3}$, Figure S16: ${ }^{13} \mathrm{C} \mathrm{NMR}$ spectrum of 3,3'-([1,1'-biphenyl]-2,2'-diylbis(methylene))bis(1-(4-methoxyphenyl)-1H-benzo[d]imidazol3-ium) dibromide (3e) in $\mathrm{CDCl}_{3}$, Figure S17: ${ }^{1} \mathrm{H}$ NMR spectrum of $3,3^{\prime}$-([1,1'-biphenyl $]-2,2^{\prime}$-diylbis (methylene))bis(1-mesityl-1H-benzo[d]imidazol-3-ium) dibromide (3f) in $\mathrm{CDCl}_{3}$, Figure S18: ${ }^{13} \mathrm{C}$ NMR spectrum of 3,3'-([1,1'-biphenyl]-2,2' -diylbis(methylene))bis(1-mesityl-1H-benzo[d]imidazol-3ium) dibromide (3f) in $\mathrm{CDCl}_{3}$, Figure S19: ${ }^{1} \mathrm{H}$ NMR spectrum of 4-acetylbiphenyl (6aa) in $\mathrm{CDCl}_{3}$, Figure S20: ${ }^{13} \mathrm{C}$ NMR spectrum of 4-acetylbiphenyl (6aa) in $\mathrm{CDCl}_{3}$, Figure S21: ${ }^{1} \mathrm{H}$ NMR spectrum of 3-acetylbiphenyl (6ba) in $\mathrm{CDCl}_{3}$, Figure S22: ${ }^{13} \mathrm{C}$ NMR spectrum of 3-acetylbiphenyl (6ba) in $\mathrm{CDCl}_{3}$, Figure S23: ${ }^{1} \mathrm{H}$ NMR spectrum of 2-acetylbiphenyl (6ca) in $\mathrm{CDCl}_{3}$, Figure S24: ${ }^{13} \mathrm{C}$ NMR spectrum of 2-acetylbiphenyl (6ca) in $\mathrm{CDCl}_{3}$, Figure S25: ${ }^{1} \mathrm{H}$ NMR spectrum of $4^{\prime}$-phenylpropiophenone (6da) in $\mathrm{CDCl}_{3}$, Figure S26: ${ }^{13} \mathrm{C}$ NMR spectrum of $4^{\prime}$-phenylpropiophenone (6da) in $\mathrm{CDCl}_{3}$, Figure S27: ${ }^{1} \mathrm{H}$ NMR spectrum of $3^{\prime}$-phenylpropiophenone (6ea) in $\mathrm{CDCl}_{3}$, Figure S28: ${ }^{13} \mathrm{C} \mathrm{NMR}$ spectrum of $3^{\prime}$-phenylpropiophenone (6ea) in $\mathrm{CDCl}_{3}$, Figure S29: ${ }^{1} \mathrm{H}$ NMR spectrum of 4-nitrobiphenyl (6fa) in $\mathrm{CDCl}_{3}$, Figure S30: ${ }^{13} \mathrm{C}$ NMR spectrum of 4-nitrobiphenyl (6fa) in $\mathrm{CDCl}_{3}$, Figure S31: ${ }^{1} \mathrm{H}$ NMR spectrum of 2-nitrobiphenyl (6ga) in $\mathrm{CDCl}_{3}$, Figure S32: ${ }^{13} \mathrm{C} \mathrm{NMR}$ spectrum of 2-nitrobiphenyl (6ga) in $\mathrm{CDCl}_{3}$, Figure S33: ${ }^{1} \mathrm{H}$ NMR spectrum of 2,4-dinitrobiphenyl (6ha) in $\mathrm{CDCl}_{3}$, Figure S34: ${ }^{13} \mathrm{C}$ NMR spectrum of 2,4-dinitrobiphenyl (6ha) in $\mathrm{CDCl}_{3}$, Figure S35: ${ }^{1} \mathrm{H}$ NMR spectrum of 4cyanobiphenyl (6ia) in $\mathrm{CDCl}_{3}$, Figure S36: ${ }^{13} \mathrm{C}$ NMR spectrum of 4-cyanobiphenyl (6ia) in $\mathrm{CDCl}_{3}$, Figure S37: ${ }^{1} \mathrm{H}$ NMR spectrum of diphenylmethane (6ja) in $\mathrm{CDCl}_{3}$, Figure S38: ${ }^{13} \mathrm{C} \mathrm{NMR} \mathrm{spec-}$ trum of diphenylmethane (6ja) in $\mathrm{CDCl}_{3}$, Figure S39: ${ }^{1} \mathrm{H}$ NMR spectrum of 4-methylbiphenyl (6ka) in $\mathrm{CDCl}_{3}$, Figure S40: ${ }^{13} \mathrm{C}$ NMR spectrum of 4-methylbiphenyl (6ka) in $\mathrm{CDCl}_{3}$, Figure S41: ${ }^{1} \mathrm{H}$ NMR spectrum of 3-methylbiphenyl (6la) in $\mathrm{CDCl}_{3}$, Figure S42: ${ }^{13} \mathrm{C}$ NMR spectrum of 3methylbiphenyl (6la) in $\mathrm{CDCl}_{3}$, Figure S43: ${ }^{1} \mathrm{H}$ NMR spectrum of 2-methylbiphenyl (6ma) in $\mathrm{CDCl}_{3}$, Figure S44: ${ }^{13} \mathrm{C}$ NMR spectrum of 2-methylbiphenyl (6ma) in $\mathrm{CDCl}_{3}$, Figure S45: ${ }^{1} \mathrm{H}$ NMR spectrum of 1-(4-(naphthalen-1-yl)phenyl)propan-1-one $(\mathbf{6 d b})$ in $\mathrm{CDCl}_{3}$, Figure $\mathrm{S} 46$ : ${ }^{13} \mathrm{C}$ NMR spectrum of 1-(4-(naphthalen-1-yl)phenyl)propan-1-one (6db) in $\mathrm{CDCl}_{3}$, Figure S47: ${ }^{1} \mathrm{H}$ NMR spectrum of 1-(4-nitrophenyl)naphthalene (6fb) in $\mathrm{CDCl}_{3}$, Figure S48: ${ }^{13} \mathrm{C}$ NMR spectrum of 1-(4nitrophenyl)naphthalene (6fb) in $\mathrm{CDCl}_{3}$, Figure S49: ${ }^{1} \mathrm{H}$ NMR spectrum of 4-(1-naphthyl)benzonitrile (6ib) in $\mathrm{CDCl}_{3}$, Figure S50: ${ }^{13} \mathrm{C}$ NMR spectrum of 4-(1-naphthyl)benzonitrile (6ib) in $\mathrm{CDCl}_{3}$, Figure S51: ${ }^{1} \mathrm{H}$ NMR spectrum of 1-(3-(naphthalen-1-yl)phenyl)propan-1-one (6eb) in $\mathrm{CDCl}_{3}$, Figure S52: ${ }^{13} \mathrm{C}$ NMR spectrum of 1-(3-(naphthalen-1-yl)phenyl)propan-1-one (6eb) in $\mathrm{CDCl}_{3}$, Figure S53: ${ }^{1} \mathrm{H} \mathrm{NMR}$ spectrum of 1-benzylnapthalene $(\mathbf{6 j b})$ in $\mathrm{CDCl}_{3}$, Figure S54: ${ }^{13} \mathrm{C}$ NMR spectrum of 1-benzylnapthalene $(6 \mathbf{j b})$ in $\mathrm{CDCl}_{3}$, Figure S55: ${ }^{1} \mathrm{H}$ NMR spectrum of 1-(2-nitrophenyl)naphthalene $(6 \mathbf{g b})$ in $\mathrm{CDCl}_{3}$, Figure S56: ${ }^{13} \mathrm{C}$ NMR spectrum of 1-(2-nitrophenyl)naphthalene $(\mathbf{6 g b})$ in $\mathrm{CDCl}_{3}$, Figure S57: ${ }^{1} \mathrm{H}$ NMR spectrum of 1-(2,4-dinitrophenyl)naphthalene $(6 \mathbf{h b})$ in $\mathrm{CDCl}_{3}$, Figure $558:{ }^{13} \mathrm{C}$ NMR spectrum of 1-(2,4-dinitrophenyl)naphthalene (6hb) in $\mathrm{CDCl}_{3}$.

Author Contributions: Conceptualization, D.-S.L.; methodology, T.W. and S.-J.H.; validation, T.W., T.-R.W., S.-J.H. and Y.-T.L.; formal analysis, T.W. and S.-J.H.; investigation, T.W., T.-R.W., S.-J.H. and Y.-T.L.; data curation, T.W., T.-R.W. and Y.-T.L.; writing-original draft preparation, D.-S.L.; writing-review and editing, T.-J.L.; supervision, D.-S.L.; project administration, D.-S.L.; funding acquisition, D.-S.L. All authors have read and agreed to the published version of the manuscript.

Funding: This research was funded by the Ministry of Science and Technology of the Republic of China, grant number 107WFA0510613.

Data Availability Statement: Data is contained within the article or Supplementary Materials. 
Acknowledgments: We thank to National Center for High-performance Computing (NCHC) for providing computational and storage resources, the Instrument Center of National Chung Hsing University for help with measurements of the high-resolution mass spectrometer, and Dao-Wen Luo for help with the X-ray analysis.

Conflicts of Interest: The authors declare no conflict of interest.

\section{References}

1. Miyaura, N.; Suzuki, A. Palladium-Catalyzed Cross-Coupling Reactions of Organoboron Compounds. Chem. Rev. 1995, 95, 2457-2483. [CrossRef]

2. Stanforth, S.P. Catalytic Cross-Coupling Reactions in Biaryl Synthesis. Tetrahedron 1998, 54, 263-303. [CrossRef]

3. Suzuki, A. Recent advances in the cross-coupling reactions of organoboron derivatives with organic electrophiles, 1995-1998. J. Organomet. Chem. 1999, 576, 147-168. [CrossRef]

4. Mingji, D.; Liang, B.; Wang, C.; You, Z.; Xiang, J.; Dong, G.; Chen, J.; Yang, Z. A Novel Thiourea Ligand Applied in the Pd-Catalyzed Heck, Suzuki and Suzuki Carbonylative Reactions. Adv. Synth. Catal. 2004, 346, 1669-1673. [CrossRef]

5. Suzuki, A.; Yamamoto, Y. Cross-coupling Reactions of Organoboranes: An Easy Method for C-C Bonding. Chem. Lett. 2011, 40, 894-901. [CrossRef]

6. Grushin, V.V.; Alper, H. Transformations of Chloroarenes, Catalyzed by Transition-Metal Complexes. Chem. Rev. 1994, 94, 1047-1062. [CrossRef]

7. Littke, A.F.; Fu, G.C. Palladium-Catalyzed Coupling Reactions of Aryl Chlorides. Angew. Chem. Int. Ed. 2002, 41, 4176-4211. [CrossRef]

8. Corbet, J.-P.; Mignani, G. Selected Patented Cross-Coupling Reaction Technologies. Chem. Rev. 2006, 106, 2651-2710. [CrossRef]

9. Wong, S.M.; Yuen, O.Y.; Choy, P.Y.; Kwong, F.Y. When Cross-Coupling Partners Meet Indolylphosphines. Coord. Chem. Rev. 2015, 293-294, 158-186. [CrossRef]

10. Schwarz, J.B.; Böhm, V.P.W.; Gardiner, M.G.; Grosche, M.; Hermann, W.A.; Hieringer, W.; Raudaschl-Sieber, G. Polymer-Supported Carbene Complexes of Palladium: Well-Defined, Air-Stable, Recyclable Catalysts for the Heck Reaction. Chem. Eur. J. 2000, 6, 1773-1780. [CrossRef]

11. Hermann, W.A.; Öfele, K.; Preysing, D.; Herdtweck, E. Metal complexes of acyclic diaminocarbenes: Links between N-heterocyclic carbene (NHC)- and Fischer-carbene complexes. J. Organomet. Chem. 2003, 684, 235-248. [CrossRef]

12. Herrmann, W.A.; Reisinger, C.-P.; Spiegler, M. Chelating N-heterocyclic carbene ligands in palladium-catalyzed heck-type reactions. J. Organomet. Chem. 1998, 557, 93-96. [CrossRef]

13. Zhang, C.; Trudell, M.L. Palladium-bisimidazol-2-ylidene complexes as catalysts for general and efficient Suzuki cross-coupling reactions of aryl chlorides with arylboronic acids. Tetrahedron Lett. 2000, 41, 595-598. [CrossRef]

14. Özdemir, İ.; Çetinkaya, B.; Demir, S.; Gürbüz, N. Palladium-catalyzed Suzuki-Miyaura reaction using saturated N-heterocyclic ligands. Catal. Lett. 2004, 97, 37-40. [CrossRef]

15. Özdemir, İ.; Gök, Y.; Gürbüz, N.; Çetinkaya, E.; Çetinkaya, B. Suzuki-Miyaura Reaction of Unactivated Aryl Chlorides Using Beznimidazol-2-Ylidene Ligands. Synth. Commun. 2004, 34, 4135-4144. [CrossRef]

16. Özdemir, İ.; Gök, Y.; Gürbüz, N.; Çetinkaya, E.; Çetinkaya, B. Palladium-Catalyzed Suzuki Reaction Using 1,3-Dialkylbenzimidazol2-ylidene Ligands in Aqueous Media. Heteroat. Chem. 2004, 15, 419-423. [CrossRef]

17. Shi, M.; Qian, H.-X. A new dimeric bidentated NHC-Pd(II) complex from trans-cyclohexane-1,2-diamine for Suzuki reaction and Heck reaction. Tetrahedron 2005, 61, 4949-4955. [CrossRef]

18. Xu, Q.; Duan, W.-L.; Lei, Z.-Y.; Zhu, Z.-B.; Shi, M. A novel cis-chelated Pd(II)-NHC complex for catalyzing Suzuki and Heck-type cross-coupling reactions. Tetrahedron 2005, 61, 11225-11229. [CrossRef]

19. Demir, S.; Özdemir, İ.; Çetinkaya, B. Use of bis(benzimidazolium)-palladium system as a convenient catalyst for Heck and Suzuki coupling reactions of aryl bromides and chlorides. Appl. Organometal. Chem. 2006, 20, 254-259. [CrossRef]

20. Özdemir, I.; Demir, S.; Çetinkaya, B. Novel tetrahydropyrimidinium/palladium system as a convenient catalysts: Suzuki coupling reactions of aryl chlorides. Arkivoc 2007, 13, 71-78. [CrossRef]

21. Liu, Z.; Zhang, T.; Shi, M. Cyclometalated cis-Chelated Bidentate N-Heterocyclic Carbene Palladium Complexes: Synthetic, Structural, and Catalytic Studies. Organometallics 2008, 27, 2668-2671. [CrossRef]

22. Avery, K.B.; Devine, W.G.; Kormos, C.M.; Leadbeater, N.E. Use of a silicon carbide multi-well plate in conjunction with microwave heating for rapid ligand synthesis, formation of palladium complexes, and catalyst screening in a Suzuki coupling. Tetrahedron Lett. 2009, 50, 2851-2853. [CrossRef]

23. Yilmaz, U.; Şireci, N.; Deniz, S.; Küçükbay, H. Synthesis and microwave-assisted catalytic activity of novel bis-benzimidazoles salts bearing furfuryl and thenyl moieties in Heck and Suzuki cross-coupling reactions. Appl. Organometal. Chem. 2010, 24, 414-420.

24. Micksch, M.; Strassner, T. Palladium(II) Complexes with Chelating Biscarbene Ligands in the Catalytic Suzuki-Miyaura CrossCoupling Reaction. Eur. J. Org. Chem. 2012, 5872-5880. [CrossRef]

25. Li, Y.; Tang, J.; Gu, J.; Wang, Q.; Sun, P.; Zhang, D. Chiral 1,2-Cyclohexane-Bridged Bis-NHC Palladium Catalysts for Asymmetric Suzuki-Miyaura Coupling: Synthesis, Characterization, and Steric Effects on Enantiocontrol. Organometallics 2014, 33, 876-884. [CrossRef] 
26. Charbonneau, M.; Addoumieh, G.; Oguadinma, P.; Schmitzer, A.R. Support-Free Palladium-NHC Catalyst for High Recyclable Heterogeneous Suzuki-Miyaura Coupling in Neat Water. Organometallics 2014, 33, 6544-6549. [CrossRef]

27. Thapa, R.; Kilyanek, S.M. Synthesis and structural characterization of 20-membered macrocyclic rings bearing trans-chelating bis(N-heterocyclic carbene) ligands and the catalytic activity of their palladium(II) complexes. Dalton Trans. 2019, 48, 12577-12590. [CrossRef]

28. Lin, Y.-R.; Chiu, C.-C.; Chiu, H.-T.; Lee, D.-S.; Lu, T.-J. Bis-benzimidazolium-palladium system catalyzed Suzuki-Miyaura coupling reaction of aryl bromides under mild conditions. Appl. Organometal. Chem. 2017, 32, e3896. [CrossRef]

29. Chiu, C.-C.; Chiu, H.-T.; Lee, D.-S.; Lu, T.-J. An efficient class of bis-NHC salts: Applications in Pd-catalyzed reactions under mild reaction conditions. RSC Adv. 2018, 8, 26407-26415. [CrossRef]

30. Tran, V.M.; Nguyen, T.K.N.; Sorna, V.; Loganathan, D.; Kuberan, B. Synthesis and Assessment of Glycosaminoglycan Priming Activity of Cluster-xylosides for Potential Use as Proteoglycan Mimetics. ACS Chem. Biol. 2013, 8, 949-957. [CrossRef]

31. Yu, K.-K.; Li, K.; Hou, J.-T.; Yu, X.-Q. Coumarin-TPA Derivative: A reaction-based ratiometric fluorescent probe for Cu(I). Tetrahedron Lett. 2013, 54, 5771-5774. [CrossRef]

32. Cervantes-Reyes, A.; Rominger, F.; Rudolph, M.; Hashmi, A.S.K. Gold(I) Complexes Stabilized by Nine- and Ten-Membered N-Heterocyclic Carbene Ligands. Chem. Eur. J. 2019, 25, 11745-11757. [CrossRef] [PubMed]

33. Liu, H.; Fridman, N.; Tamm, M.; Eisten, M.S. Addition of E-H (E = N, P, C, O, S) Bonds to Heterocumulenes Catalyzed by Benzimidazolin-2-iminato Actinide Complexes. Organometallics 2017, 36, 3896-3903. [CrossRef]

34. Chan, A.; Scheidt, K.A. Highly Stereoselective Formal [3+3] Cycloaddition of Enals and Azomethine Imines Catalyzed by N-Heterocyclic Carbenes. J. Am. Che. Soc. 2007, 129, 5334-5335. [CrossRef] [PubMed]

35. Glannerini, M.; Vila, C.; Hornillos, V.; Feringa, B.L. One-Pot, Fast and Modular Approach to Alkyl- and Aryl Ketones via Sequential 1,2-Addition,Pd-Catalyzed Cross-Coupling of Organolithium Reagents with Weinreb Amides. Chem. Commun. 2016, 52, 1206-1209. [CrossRef]

36. Liu, Z.; Tan, H.; Wang, L.; Fu, T.; Xia, Y.; Zhang, Y.; Wang, J. Transition-Metal-Free Intramolecular Carbene Aromatic Substitution/Büchner Reaction: Synthesis of Fluorenes and [6,5,7]Benzo-fused Rings. Angew. Chem. Int. Ed. 2015, 54, 3056-3060. [CrossRef]

37. Mao, P.; Yang, L.; Xiao, Y.; Yuan, J.; Liu, X.; Song, M. Suzuki cross-coupling catalyzed by palladium(II) complexes bearing 1-aryl-3,4,5,6-tetrahydropyrimidine ligands. J. Organomet. Chem. 2012, 705, 39-43. [CrossRef]

38. Chen, L.; Lang, H.; Fang, L.; Yu, J.; Wang, L. Nickel-Catalyzed Desulfitative Suzuki-Miyaura Cross-Coupling of N,NDisulfinylmethylamines and Arylboromic Acids. Eur. J. Org. Chem. 2014, 2014, 6385-6389. [CrossRef]

39. Han, C.; Zhang, Z.; Xu, S.; Wang, K.; Chen, K.; Zhao, J. Palladium-Catalyzed Hiyama Coupling of Benzylic Ammonium Salts via C-N Bond Cleavage. J. Org. Chem. 2019, 84, 16308-16313. [CrossRef]

40. Piontek, A.; Ochędzan-Siodłak, W.; Bisz, E.; Szostak, M. Nickel-Catalyzed C(sp $\left.{ }^{2}\right)-\mathrm{C}\left(\mathrm{sp}^{3}\right)$ Kumada Cross-Coupling of Aryl Tosylates with Alkyl Grignard Reagents. Adv. Synth. Catal. 2019, 361, 2329-2335. [CrossRef]

41. Liu, H.; Yin, B.; Gao, Z.; Li, Y.; Jiang, H. Transition-metal-free highly chemo- and regioselective arylation of unactivated arenes with aryl halides over recyclable heterogeneous catalysts. Chem. Commun. 2012, 48, 2033-2035. [CrossRef] [PubMed]

42. Lücke, A.-L.; Wlechmann, S.; Freese, T.; Schmidt, A. Suzuki-Miyaura Cross-Coupling Reactions in Acetic Acid Employing Sydnone-Derived Catalyst Systems. Synlett 2017, 28, 1990-1993.

43. Song, B.; Knauber, T.; Gooßen, L.J. Decarboxylative Cross-Coupling of Mesylates Catalyzed by Copper/Palladium Systems with Customized Imidazolyl Phosphine Ligands. Angew. Chem. Int. Ed. 2013, 52, 2954-2958. [CrossRef] [PubMed] 